\title{
Exploring Topological Superconductivity in Topological Materials
}

\author{
Yupeng $\mathrm{Li}^{1}$ and $\mathrm{Zhu}-\mathrm{An} \mathrm{Xu}^{1,2,3, \text { * }}$ \\ ${ }^{1}$ Zhejiang Province Key Laboratory of Quantum Technology and Device, \\ Department of Physics, Zhejiang University, Hangzhou 310027, China \\ ${ }^{2}$ Zhejiang California International NanoSystems Institute, Zhejiang University, Hangzhou 310058, China \\ ${ }^{3}$ Collaborative Innovation Centre of Advanced Microstructures, Nanjing University, Nanjing 210093, China
}

(Dated: July 11, 2019)

\begin{abstract}
The exploration of topological superconductivity and Majorana zero modes has become a rapidly developing field. Many types of proposals to realize topological superconductors have been presented, and significant advances have been recently made. In this review, we conduct a survey on the experimental progress in possible topological superconductors and induced superconductivity in topological insulators or semimetals as well as artificial structures. The approaches to inducing superconductivity in topological materials mainly include high pressure application, the hard-tip point contact method, chemical doping or intercalation, the use of artificial topological superconductors, and electric field gating. The evidence supporting topological superconductivity and signatures of Majorana zero modes are also discussed and summarized.
\end{abstract}

\section{INTRODUCTION}

Topological superconductors (TSCs) with protected Majorana surface states have become a focus of interest due to their potential applications in the fault-tolerant topological quantum computation! 1] Topological classifications of gapped quantum many-body systems have sparked a revolution in condensed matter physics. Nontrivial topology in relation to superfluid helium $3\left({ }^{3} \mathrm{He}\right)$ was discussed by Volovik in $2003, \frac{2}{2}$ and topologically nontrivial superconductors were introduced approximately two decades ago in a two-dimensional (2D) model by Read and Green ${ }^{3}$ as well as in a one-dimensional (1D) model by Kitaev ${ }^{4}$ A non-Abelian Majorana zero mode in the vortex core in the $2 \mathrm{D}$ case or at the edge in the $1 \mathrm{D}$ case was theoretically predicted in these works, long before the recent research boom in the field of topological phases of matter.

Majorana fermions, whose particles are their own antiparticles, were first introduced by Ettore Majorana in the context of elementary particle physics ${ }^{[5}$ Unlike in particle physics, in condensed matter physics, if the creation operator $\gamma^{+}$of a quasiparticle, which is a superposition of electron and hole excitations, is the same as the annihilation operator $\gamma$, then this type of a particle can be regarded as a Majorana fermion. In the Read-Green model,$\stackrel{[3}{a}$ the Bogoliubov quasiparticles (neutral excitations in superconductors) in the bulk can be identified as dispersive Majorana fermions, which can be bound to a defect at zero energy, and these combined objects are called Majorana bound states or Majorana zero modes $(\mathrm{MZMs}) \cdot{ }^{6 / 77}$ MZMs localized at defects have been theoretically predicted to obey exotic exchange statistics, similar to non-Abelian anyons, which will change their quantum states if they are interchanged. This phenomenon is the most attractive property of MZMs. Exchanging the posi-

\footnotetext{
* zhuan@zju.edu.cn
}

tion of MZMs corresponds to a nontrivial transformation within the degenerate ground-state manifold and represents a noncommutative operation, which does not depend on the method or details of the execution. Such an operation is topologically protected and only depends on the exchange statistics of quasiparticles; thus, it can be used in quantum gates or topological quantum computing.

Unfortunately, little progress was made in realizing TSCs before the discovery of topological insulators (TIs). Intrinsic TSCs are rare because they require odd-parity pairing, and such pairing states usually require a particular combination of pairing interactions and electronic structure. A conceptual breakthrough in the context of TIs emerged from the seminal work of $\mathrm{Fu}$ and Kane ${ }^{819}$ Their theory predicts that surface Dirac fermions of TIs may realize topological superconductivity when in proximity to an s-wave superconductor ${ }^{10 \mid 11}$ Subsequently, Fu and Berg further propose a new mechanism for oddparity pairing facilitated by strong spin-orbit coupling (SOC) such that time-reversal-invariant TSCs can be realized with carrier-doped TIs such as $\mathrm{Cu}_{x} \mathrm{Bi}_{2} \mathrm{Se}_{3}{ }^{12}$ Theoretical proposals on the realization of MZMs in hybrid systems of s-wave superconductors deposited on the surface of a strong TI and related structures, ${ }^{7 / 10 \mid 13}$ such as semiconducting nanowires, have motivated intense efforts to 'engineer' topological superconducting states through the simple building of components.

Strong TSCs have three characteristics:11 (1) oddparity pairing symmetry with a full superconducting gap; (2) gapless surface states consisting of Majorana fermions; and (3) MZMs in the superconducting vortex cores. In addition, unconventional superconductors usually have nodal superconducting gaps. If the nodes themselves have topological properties, such unconventional superconductors can also be regarded as TSCs, and they are called weak TSCs! 14

Generally, TSCs can also be classified as time-reversalbreaking TSCs and time-reversal-invariant TSCs according to the presence or absence of internal symme- 
tries (such as time reversal symmetry and spin rotation symmetry) $\frac{15}{15}$ respectively. $\mathrm{Sr}_{2} \mathrm{RuO}_{4}$ is often regarded as a typical example of the former type. The existence of an internal magnetic field in the superconducting state that breaks the time reversal symmetry has been supported by nuclear magnetic resonance (NMR) measurements $\frac{16}{16}$ and muon spin rotation $(\mu \mathrm{SR})$ experiments $\frac{17118}{1}$ The other approach to achieving time-reversal-breaking TSCs is to fabricate semiconductor/superconductor heterostructures in which the MZMs are located at the ends of the semiconductor nanowire $\frac{19}{9}$ The latter type, as a new type of TCS, requires strong SOC to induce oddparity pairing. $\mathrm{Cu}_{x} \mathrm{Bi}_{2} \mathrm{Se}_{3}$ is proposed as a promising candidate for this type ${ }^{12}$ Moreover, the proximity effect in TI/superconductor heterostructures will also induce time-reversal-invariant TCSs in which the MZMs could exist at the vortex cores. Usually, MZMs can be characterized by the zero-bias peak (ZBP) in the differential conductance obtained through scanning tunneling microscopy (STM) measurements.20121 Other features of these TCSs, such as gapless surface states, can be detected by measuring nontrivial transport properties, for instance, a $4 \pi$-periodic Josephson supercurrent ${ }^{22123}$ and helical edge states in the supercurrent. ${ }^{24}$

A variety of routes can be followed to realize topological superconductivity, and many experimental reports about confirming MZMs have been presented. This review mainly focuses on the superconductivity induced in materials with nontrivial topology as well as the superconductivity arising through the proximity effect in 'hybrid systems', and the current situation of pristine TSCs is also briefly summarized. In section I, we will first introduce the TSC candidates among pristine superconductors, such as $\mathrm{Sr}_{2} \mathrm{RuO}_{4}, \mathrm{UPt}_{3}$, and iron-based superconductors with nontrivial topological features. In section II, the high pressure technique is introduced as a common method to realize superconductivity in topological materials, such as TIs, Dirac semimetals (DSMs), and Weyl semimetals (WSMs). However, performing further experiments to observe the pairing states and gapless surface states under pressure is quite difficult. In section III, the hard-tip point contact method, similar to the high pressure technique, provides a new platform to obtain superconductivity in topological materials. One advantage of this technique is that the Andreev reflection can be measured to detect MZMs. In section IV, superconductivity induced in topological materials by either doping or intercalation is summarized, which is certainly worth further investigating. In section $\mathrm{V}$, artificial structures, such as nanowire/superconductor heterostructures and TI/superconductor heterostructures, are introduced, which are the most successful approaches to realizing TSCs, and very strong signatures of MZMs are presented. Moreover, an electric field gating technique is also introduced because it is widely used in the measurement of artificial structures. Finally, a summary and an outlook on this field are presented.

\section{PRISTINE TOPOLOGICAL SUPERCONDUCTORS}

To date, most superconductors are s-wave superconductors with spin-singlet pairing, and such conventional s-wave pristine superconductors are clearly nontopological because they exhibit a smooth crossover from the weak-coupling Bardeen-Cooper-Schrieffer (BCS) limit to the strong-coupling Bose-Einstein condensate (BEC) limit without undergoing a gap-closing phase transition. Namely, we should search for TSCs by studying superconductors with unconventional pairing symmetry. We know that the total spin $(S)$ of a Cooper pair has two states ${ }^{25}$ the spin-singlet pairing state with $S=0$ and the spin-triplet pairing state with $S=1$. Therefore, odd parity of the wave function of a Cooper pair $(S=0)$ requires even parity of the orbital wave function $(L=$ $0,2,4 \ldots)$, satisfying opposite parity of the total wave function. Moreover, $L=0$ or 2 corresponds to s-wave or d-wave pairing, respectively. In spin-triplet states $(S=$ 1 ), the orbital wave function should be odd parity $[L=$ 1 (p-wave), 3 ( $f$-wave), $5 \ldots$...

In general, a majority of superconductors are known to exhibit spin-singlet pairing states, such as conventional superconductors, cuprate superconductors,$\frac{26}{}$ and ironbased superconductors ${ }^{27}$ Therefore, to search for TSCs, time-reversal-symmetry-breaking superconductors with spin-triplet pairing states have attracted tremendous attention, although they are quite rare. If pristine superconductors with unconventional pairing symmetry have nontrivial topology, they can be called 'intrinsic' TSCs. A relatively small number of candidates for spin-triplet superconductors exist. Several candidates, based on substantial evidence, may possess spin-triplet pairing, but none has been as firmly confirmed as superfluid ${ }^{3} \mathrm{He}$. The promising candidates for spin-triplet pairing are $\mathrm{Sr}_{2} \mathrm{RuO}_{4}$ and $\mathrm{UPt}_{3}$, although their superconducting transition temperature $\left(T_{c}\right)$ is low. Other possible spin-triplet superconductors include ferromagnetic heavy fermion superconductors, such as $\mathrm{UGe}_{2}$ and $\mathrm{UCoGe}$, and noncentrosymmetric (lacking inversion symmetry) superconductors, such as $\mathrm{Li}_{2} \mathrm{Pt}_{3} \mathrm{~B}$. ${ }^{28}$ The recently discovered $\mathrm{K}_{2} \mathrm{Cr}_{3} \mathrm{As}_{3}$ family with a quasi-one-dimensional (quasi1D) structure may also have an unconventional pairing state ${ }^{29}$ In the following subsections, we will present the updated progress in the $\mathrm{Sr}_{2} \mathrm{RuO}_{4}, \mathrm{UPt}_{3}{ }^{14}$ and iron-based superconductors. Regarding the $\mathrm{K}_{2} \mathrm{Cr}_{3} \mathrm{As}_{3}$ family, little progress has been made in terms of their pairing states due to their extremely air-sensitive properties.

\section{A. $\mathrm{Sr}_{2} \mathrm{RuO}_{4}$}

Stimulated by the discovery of the high $T_{c}$ cuprate superconductors $\mathrm{La}_{2-x} \mathrm{Ba}_{x} \mathrm{CuO}_{4}, \frac{30}{}$ the element substitution method has been widely employed for perovskitetype tetragonal crystal structures to search for new high $T_{c}$ superconductors, and a large family of high $T_{c}$ cuprate 
superconductors has emerged. Many attempts to replace copper with other $3 \mathrm{~d}$ transition metals have been made, which finally led to the discovery of a new interesting superconductor, $\mathrm{Sr}_{2} \mathrm{RuO}_{4}$, with $T_{c} \approx 1 \mathrm{~K}$ in $1994^{31}$ This new superconductor has several unusual properties, such as an impurity-sensitive $T_{c}$, absence of a coherence peak in the NMR spectrum, and a linear specific heat at low temperature 32

The density of states at the Fermi surface in normal metals can generate a paramagnetic susceptibility $\chi_{p}$, i.e., the Pauli susceptibility, and a magnetic field $(B)$ can lower the free energy by $\frac{1}{2} \chi_{p} B^{2}{ }^{[33}$ However, superconductors with spin-singlet pairing states would suppress paramagnetic susceptibility at absolute temperature because no electron exists at the Fermi surface and Cooper pairs form instead. Therefore, spin susceptibility disappears in the spin-singlet pairing states. However, the spin-triplet state allows paramagnetic susceptibility, and this characteristic can be employed as an experimental clue to spintriplet superconductivity. NMR is an effective method to measure the spin states of electrons. The Knight shift related to the spin susceptibility was reported to remain unchanged in $\mathrm{Sr}_{2} \mathrm{RuO}_{4}$ when the temperature is below $T_{c}{ }^{[16}$ In addition, the time-reversal-symmetry-breaking superconductors exhibit an internal magnetic field below $T_{c}$ due to the spin-triplet pairing states, which has also been successfully detected by $\mu \mathrm{SR}$ experiments 17

Another key experiment to support this spin-triplet state of $\mathrm{Sr}_{2} \mathrm{RuO}_{4}$ is phase-sensitive measurements $\stackrel{34}{\text { For }}$ such studies, a superconducting quantum interference device (SQUID) with a Josephson junction of $\mathrm{Au}_{0.5} \mathrm{In}_{0.5^{-}}$ $\mathrm{Sr}_{2} \mathrm{RuO}_{4}$ located on the opposite sides of the $\mathrm{Sr}_{2} \mathrm{RuO}_{4}$ crystal was designed and prepared. The critical current $I_{c}$ across the Josephson junctions will be minimized if no flux $(\Phi=0)$ occurs in the SQUID according to the following formula: $34 \mid 35$

$$
I_{c} \propto \cos \left[\left(\frac{\Phi}{\Phi_{0}}+\frac{1}{2}\right) \pi\right],
$$

where $\Phi_{0}=h / 2 e$ is the flux quantum. A maximum value of $I_{c}$ is observed at $\Phi=0$ in a conventional SQUID, for example, when the two terminals of the Josephson junction are placed on the same side of the $\mathrm{Sr}_{2} \mathrm{RuO}_{4}$ crystal. In contrast, the magnetic-field-dependent critical current shows a $\pi$ phase shift in the opposite case, indicating oddparity pairing symmetry in $\mathrm{Sr}_{2} \mathrm{RuO}_{4}$. However, because of the existence of chiral domain structures in $\mathrm{Sr}_{2} \mathrm{RuO}_{4}$, the above experiments are hard to reproduce, and a direct observation of chiral domains is lacking. ${ }^{28136}$ Subsequently, this $\pi$ phase shift of a similar Josephson junction was also observed by another group $\frac{37}{}$ Chiral order parameter domains of the form $p_{x} \pm i p_{y}$ were found in these studies, which is direct evidence for complex p-wave pairing symmetry. ${ }^{37}$

The proposed pairing symmetry in $\mathrm{Sr}_{2} \mathrm{RuO}_{4}$ is similar to the A-phase of superfluid ${ }^{3} \mathrm{He}{ }^{32}$ Half-quantum fluxoids have been predicted and should be accompanied by MZMs in the vortices ${ }^{3}$ As expected, half-height magne- tization steps have been successfully observed in annular $\mathrm{Sr}_{2} \mathrm{RuO}_{4}$ samples, suggesting the existence of halfquantum fluxoids, $\frac{38}{18}$ which can give rise to MZMs. $\frac{3139}{3}$ Moreover, the differential conductance spectrum shows a broad hump, revealing another important characteristic of supercurrents spontaneously generated at edges. $\underline{40}$

The evidence of spin-triplet pairing states in $\mathrm{Sr}_{2} \mathrm{RuO}_{4}$ seems to be very strong, but open questions still remain. First, the evidence of a superconducting gap in $\mathrm{Sr}_{2} \mathrm{RuO}_{4}$ is inconclusive, although a gap node has been evidenced by measurements of the specific heat, thermal conductivity, and $1 / T_{1}$ as well as by the temperature dependence of the penetration depth. $\frac{28 \mid 32141}{A}$ recent NMR study on $\mathrm{Sr}_{2} \mathrm{RuO}_{4}$ found a substantially suppressed spin susceptibility, which could cause considerable controversy over its superconducting order paramenter $\underline{42}$. According to a theoretical work, topological edge states may exist in nodal gap superconductors, but the edge state is weaker than that in fully gapped systems. ${ }^{43}$ In addition, the SOC is not very strong in $\mathrm{Sr}_{2} \mathrm{RuO}_{4}$, which causes the topological chiral surface state to not be separated and formed by ordinary fermions instead of chiral Majorana edge states.14 Therefore, the mixed surface states increase the difficulty of detecting MZMs and gapless surface states, and applying a high magnetic field may be helpful for detecting the Majorana edge states. ${ }^{44}$ More discussions on $\mathrm{Sr}_{2} \mathrm{RuO}_{4}$ can be found in recent studies. 14 28

\section{B. $\mathrm{UPt}_{3}$}

$\mathrm{UPt}_{3}$, a heavy fermion superconductor, is also known to be a strong candidate for spin-triplet superconductors. ${ }^{14 \mid 36 / 45} \mathrm{UPt}_{3}$ has a $\mathrm{MgCd}_{3}$-type hexagonal structure with the space group $P 6_{3} / m m c$. The large specific heat coefficient $(\gamma)$ and Fermi liquid behavior of the resistivity at low temperature illustrate its strong electron-electron interaction as well as its heavy fermion features ${ }^{45}$ These heavy fermion characteristics can be verified by measuring the de Haas-van Alphen effect as well as the effective masses of different bands, ranging from approximately $15 m_{e}$ to $90 m_{e}$. Thermodynamic measurements also support Fermi liquid states in $\mathrm{UPt}_{3}$. When the temperature is below $5 \mathrm{~K}$, a weak antiferromagnetic order transition emerges along with intraplane antiferromagnetic coupling and interplane ferromagnetic coupling.

Superconductivity is observed at quite low temperatures, with $T_{c}=0.53 \mathrm{~K}$, and three different superconducting phases appear, named phase A $(0.48 \mathrm{~K}<\mathrm{T}<$ $0.53 \mathrm{~K}$, low- $B)$, phase $\mathrm{B}(T<0.48 \mathrm{~K}$, low- $B)$ and phase $\mathrm{C}(T<0.48 \mathrm{~K}$, high- $B)$. The specific heat of $\mathrm{UPt}_{3}$ also shows a second transition at $T \sim 0.48 \mathrm{~K}$, and the existence of multicomponent phase diagrams can be verified by critical magnetic field measurements and other experiments 455 The internal magnetic field in phase B has been verified by $\mu \mathrm{SR}$ measurements, $\frac{18}{18}$ similar to the case of $\mathrm{Sr}_{2} \mathrm{RuO}_{4} \cdot 17$ Moreover, the polar Kerr effect re- 
flects time reversal symmetry breaking in phase $\mathrm{B}{ }^{46} \mathrm{Al}-$ though the pairing symmetry is still under debate, spin triplet $f$-wave superconductivity is believed to occur in phase $\mathrm{B}$ 14/36/45 In addition, nodes in the superconducting gap are presented in all three phases, $\frac{45}{4}$ which is supposed to be unfavorable for TSCs 11 Angular-resolved Josephson tunneling junctions experiments can probe the existence of nodes by measuring the angular dependence of the gap, and fourfold symmetry of the gap structure has been observed for the high temperature phase, which is quite different from the results for the hexagonal crystal structure. 99 The low temperature phase seems to exhibit different superconducting gaps, thus indicating a complex order parameter in $\mathrm{UPt}_{3}$.

Theoretical work has predicted the existence of two dispersive MZMs due to the mirror chiral symmetry 50 in this nodal superconductor. This material provides another possibility for realizing TSCs with nodal superconductors 51 However, because of the radioactive element uranium in $\mathrm{UPt}_{3}$, performing experiments to detect the gapless surface states and MZMs in the superconducting vortex is difficult.

\section{Iron-based superconductors}

Iron-based superconductors have drawn extensive attention over the past ten years 52 because of its high $T_{c}$, nematic phase, orbital ordering phase, abundant antiferromagnetic phases, etc. The rich phases may be related to the multiorbital physics. Therefore, intrinsic hybridization of various bands could exist in these systems and contribute to band inversions 53154 similar to in TIs, which appeals to theorists, and nontrivial topological states in some iron-based superconductors have been proposed. $\underline{53}$

Monolayer FeSe film grown on a STO substrate possesses a very high superconducting transition temperature, with $T_{c} \sim 77 \mathrm{~K}{ }^{55}$ Intriguingly, angle-resolved photoemission spectroscopy (ARPES) measurements revealed 1D topological edge states at the $M$ point in an FeSe/STO sample, where the gap forms because of band inversion caused by SOC $54 \mid 56$ This interesting work provides an exciting opportunity to investigate the coexistence of superconductivity and topological states in ironbased superconductors. In addition, when the element Se is gradually substituted by the element Te in monolayer $\mathrm{FeTe}_{1-x} \mathrm{Se}_{x} / \mathrm{STO}$, a nontrivial topological band can be detected at the $\Gamma$ point by ARPES 57 The conduction band and valence band at the $\Gamma$ point will touch at $33 \%$ Se concentration, as predicted by theoretical calculations on single layer $\mathrm{FeTe}_{1-x} \mathrm{Se}_{x},[58$ which suggests a nontrivial band topology of monolayer $\mathrm{FeTe}_{1-x} \mathrm{Se}_{x} / \mathrm{STO}$. In addition, Dirac-cone-type spin-helical surface states have been observed in $\mathrm{FeTe}_{0.55} \mathrm{Se}_{0.45}$ through high-resolution spin-resolved photoemission spectroscopy and ARPES. Sharp ZBPs inside the vortex have also been successfully detected and are regarded as evidence of Majorana bound states ${ }^{[59}$ However, in the vortex, s-wave-like quasiparticle excitations near the zero energy have also been observed in an $\mathrm{FeTe}_{0.55} \mathrm{Se}_{0.45}$ sample ${ }^{60}$ Therefore, further experiments are required to verify the TSC behavior in $\mathrm{FeTe}_{0.55} \mathrm{Se}_{0.45}$.

The progress in the topological nature of $\mathrm{FeSe}_{1-x} \mathrm{Te}_{x}$ inspired more investigations of other iron-based superconductors. Another FeSe-based superconductor, $\mathrm{Li}_{0.84} \mathrm{Fe}_{0.16} \mathrm{OHFeSe}$, was developed, 61 in which the $\mathrm{Li}_{0.84} \mathrm{Fe}_{0.16} \mathrm{OH}$ layers are intercalated between FeSe layers. $\mathrm{Li}_{0.84} \mathrm{Fe}_{0.16} \mathrm{OHFeSe}$ can be viewed as a heavily electron-doped iron selenide superconductor, and its $T_{c}$ is greatly enhanced from the value of $8 \mathrm{~K}$ in $\mathrm{FeSe}^{62}$ to $\sim 40 \mathrm{~K}$. A full superconducting gap is obtained based on STM and penetration depth measurements. $\underline{6364}$ Subsequently, a ZBP was clearly observed in tunneling spectroscopy results, 65 which is consistent with MZMs. The Dirac-cone-type surface state was also confirmed by ARPES. An early theoretical work proposed that Carolide Gennes-Matricon (CdGM) bound states are generated in the vortex with separation energy $E=\mu \Delta^{2} / F_{F}$, where $E_{F}$ is the Fermi energy, and $\mu= \pm 1 / 2, \pm 3 / 2 \ldots$ in an swave superconductor 66 The discrete energy of the bound states in a chiral p-wave superconductor will change with $\mu=0, \pm 1, \pm 3 \ldots$, and the $\mu=0$ state is the MZM $[67$ The pairing symmetry in this system is proposed to be a bonding-antibonding s-wave state or a nodeless d-wave state, ${ }^{68}[69$ and Majorana edge states have not been observed thus far. More experimental evidence is desired to confirm the topological superconductivity.

In addition to the aforementioned superconductors, several other iron-based superconductors have also been proposed to be promising TSCs. Multiple topological phases have been observed in the 111 series $\mathrm{Li}(\mathrm{Fe}, \mathrm{Co}) \mathrm{As}$ samples by ARPES ${ }^{70} \mathrm{CaFeAs}_{2}$ is likewise predicted to be an ideal TSC system, whose structure includes CaAs and FeAs layers. The CaAs layer is a topological quantum spin Hall layer and the FeAs layer is a superconducting layer; thus, this compound is also a promising system for detecting MZMs! ${ }^{71}$ Therefore, iron-based superconductors may offer a potential platform to discover TSCs, $\stackrel{53}{ }$ which may open a new direction to study ironbased superconductors.

\section{Cr-based superconductors}

The recently discovered $\mathrm{Cr}_{3} \mathrm{As}_{3}$-based quasi-1D superconductors have attracted wide attention due to their unconventional properties. Much experimental evidence for possible spin-triplet pairing states exists. ${ }^{29} \mathrm{~K}_{2} \mathrm{Cr}_{3} \mathrm{As}_{3}$ was the first synthesized member, with $T_{c}=6.1 \mathrm{~K}, 72$ and then, $\mathrm{Rb}_{2} \mathrm{Cr}_{3} \mathrm{As}_{3}\left(T_{c}=4.8 \mathrm{~K}\right)^{73}$ and $\mathrm{Cs}_{2} \mathrm{Cr}_{3} \mathrm{As}_{3}$ $\left(T_{c}=2.2 \mathrm{~K}\right)^{74}$ were discovered. Recently, $\mathrm{Na}_{2} \mathrm{Cr}_{3} \mathrm{As}_{3}$, with $T_{c} \sim 8.6 \mathrm{~K}$, was also successfully synthesized by an ion-exchange synthesis method ${ }^{75} T_{c}$ appears to decrease with increasing radius of the $A$ ions in the $A_{2} \mathrm{Cr}_{3} \mathrm{As}_{3}$ series $(A=\mathrm{Na}, \mathrm{K}, \mathrm{Rb}, \mathrm{Cs})$. 
The upper critical field of $\mathrm{K}_{2} \mathrm{Cr}_{3} \mathrm{As}_{3}$ can reach $37 \mathrm{~T}$ at $0.6 \mathrm{~K}, \frac{76177}{\text { significantly exceeding the Pauli limit } H_{c 2}}$ $=1.84 T_{c}$, indicating an unconventional pairing state.33 Moreover, Cr-spin ferromagnetic fluctuations, as measured by NMR, emerge above $T_{c},{ }^{78 \mid 79}$ and a $\mu$ SR study showed that a weak spontaneous internal magnetic field is detected below $T_{c}, \frac{80}{}$ both of which suggest potential spin-triplet pairing states in these Cr-based superconductors. Other experiments, such as penetration depth measurements, suggest gap nodes in the superconducting gap function. 2981 Because of the air-sensitive feature of the Cr-based samples, performing further measurements to investigate their topological nature is very difficult.

\section{PRESSURE INDUCED SUPERCONDUCTIVITY}

The application of high pressure, as a clean and effective method to tune the lattice and electronic structures without inducing disorder, is usually employed to investigate phase transitions and synthesize certain compounds. ${ }^{82}$ Especially in the study of superconductors, employing high pressure has been proven to be useful. $\frac{83}{}$ The highest recorded $T_{c}$ was realized in hydrogen-containing materials by applying high pressure $84 \mid 85$ Therefore, high pressure has also been widely used to explore superconductivity in topological materials. ${ }^{82}$ Although superconductivity has been induced by high pressure in all types of topological materials, including topological (crystalline) insulators, DSMs, and WSMs, much work remains before either TSCs or MZMs can be observed with this method. Probing pairing states and gapless surface states under high pressure condition is difficult. However, exploration of topological superconductivity in topological materials using the high pressure technique is worthy of continuous endeavors.

\section{A. Topological insulators under pressure}

TSCs are fully gapped in the bulk state, similar to TIs, but have a Majorana bound state instead of the Dirac surface state in TIs. ${ }^{11}$ Many theoretical predictions and experiments have been performed to investigate $2 \mathrm{D}$ TIs, such as $\mathrm{HgTe} / \mathrm{CdTe}$ quantum well structures, ${ }^{86}$ or 2D Dirac surface states in three-dimensional (3D) TIs, such as $\mathrm{Bi}_{1-x} \mathrm{Sb}_{x},{ }^{87} \mathrm{Bi}_{2} \mathrm{Te}_{3},{ }^{88} \mathrm{Bi}_{2} \mathrm{Se}_{3}, \mathrm{Sb}_{2} \mathrm{Te}_{3}{ }^{90}$ and others 91 Based on the studies of TIs, tuning TIs such that they exhibit superconductivity by applying high pressure has become an attractive option and has been performed by many groups to discover possible TSCs.

$\mathrm{Bi}_{2} \mathrm{Te}_{3}$ has been identified as a TI based on ARPES 88 and displays a superconducting transition of approximately $3 \mathrm{~K}$ under $4 \mathrm{GPa} .^{92 \mid 93} T_{c}$ increases at approximately $8 \mathrm{GPa}$, accompanied by a structural transition from the ambient-pressure phase (a rhombohedral $R$ $3 m$ structure) to the high-pressure phase (a monoclinic
$C 2 / m$ structure) 929 According to density functional theory (DFT) calculations, the topologically nontrivial band structure and surface states still exist at $4 \mathrm{GPa}, 92$ which may induce Majorana fermions in the surface states due to the proximity effect of the bulk superconducting states. 10
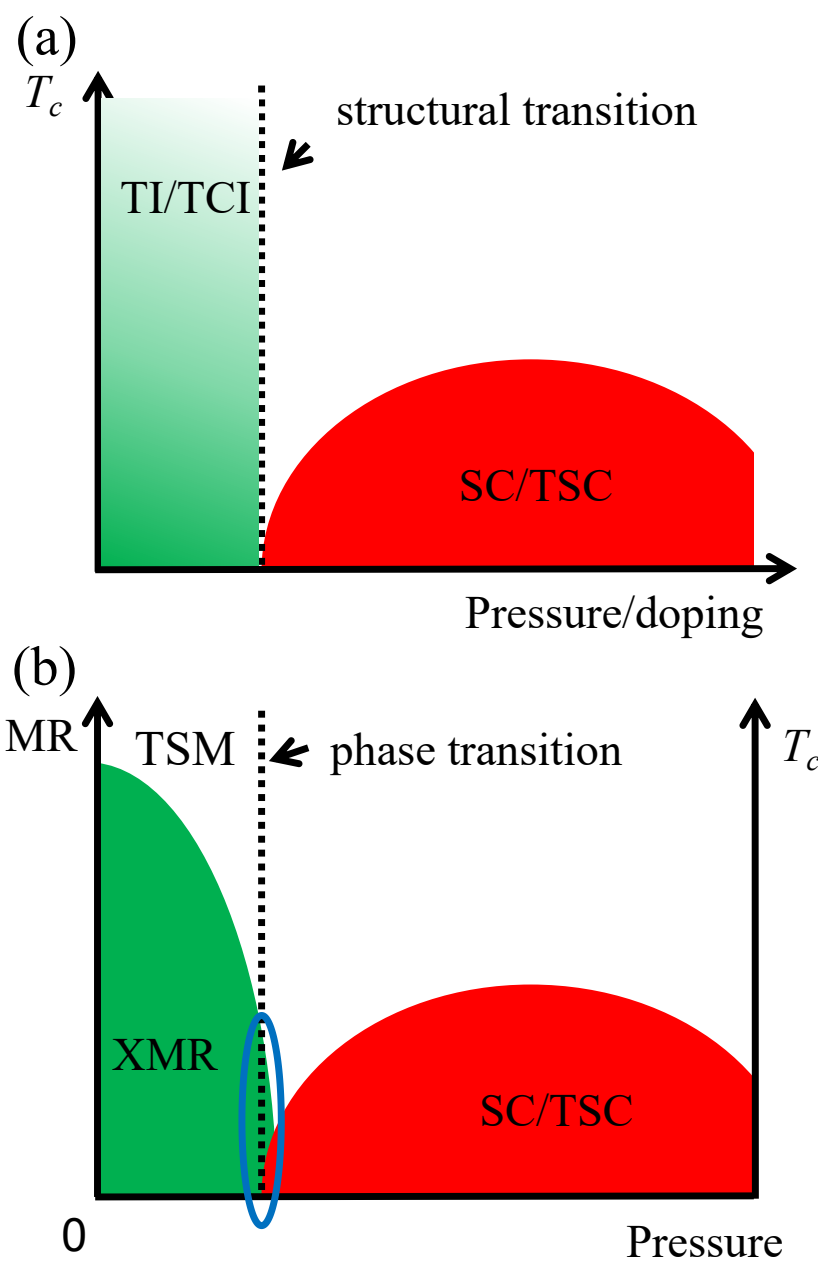

FIG. 1. Sketched phase diagrams of TIs/TCIs (a) and TSMs (b) under pressure or doping. SC, TCI, and MR represent superconductivity, topological crystalline insulator and magnetoresistance, respectively. (a) A superconducting state (red region) is usually induced by pressure after a structural transition. No structural transition emerges in the TCI/TI doping cases. For TSMs, whether the suppression of XMR or a structural transition drives the system into a superconducting state is still an open issue. XMR and SC may coexist in the region marked by the ellipse.

A more intriguing TI compound, $\mathrm{Bi}_{2} \mathrm{Se}_{3}$, could become superconducting not only through charge doping but also through high pressure application. ${ }^{95}$ When pressure is applied up to $11 \mathrm{GPa}$, a superconducting transition is successfully observed, accompanied by a structural transition from the $R-3 m$ phase to the $C 2 / m$ phase, sim- 
TABLE I. Summary of high-pressure-induced superconductivity in topological materials. Superconductivity usually appears along with a structural transition under pressure. In the $T_{c}$ column, the pressure under which superconductivity begins to emerge is listed in brackets. In the Phase II and Phase III columns, the pressure at which the structural transition begins is also listed in brackets. (In this table, TI, DSM, WSM, TSM and TPSM represent topological insulator, Dirac semimetal, Weyl semimetal, topological semimetal and triply degenerate nodal point semimetal, respectively. Pressure and temperature are simplified as $\mathrm{P}$ and $\mathrm{T}$, respectively.)

\begin{tabular}{|c|c|c|c|c|c|}
\hline Material & Type & $T_{c}$ & Phase I (Ambient P) & Phase II & Phase III \\
\hline $\mathrm{Bi}_{2} \mathrm{Se}_{3}$ & TI & $0.3-7 \mathrm{~K}(11.9 \mathrm{GPa})$ & $R-3 m$ & $C 2 / m(10 \mathrm{GPa})$ & bcc-like C2/m (28 GPa) \\
\hline $\mathrm{Bi}_{2} \mathrm{Te}_{3} 92$ & $\mathrm{TI}$ & $2.6-9.5 \mathrm{~K}(\sim 3 \mathrm{GPa})$ & $R-3 m$ & $C 2 / m(\sim 8 \mathrm{GPa})$ & - \\
\hline $\mathrm{Sb}_{2} \mathrm{Te}_{3} \sqrt{96 \mid 97}$ & $\mathrm{TI}$ & $3-7.3 \mathrm{~K}(4 \mathrm{GPa})$ & $R-3 m$ & $C 2 / m(9.3 \mathrm{GPa})$ & Im-3m $(19.8 \mathrm{GPa})$ \\
\hline BiTel ${ }^{98}$ & TI & $\sim 1-5.2 \mathrm{~K}(\sim 10 \mathrm{GPa})$ & $P 3 m 1$ & Pnma (8.8 GPa) & $P 4 / n m m(18.9 \mathrm{GPa})$ \\
\hline $\mathrm{BiTeBr}$ & $\mathrm{TI}$ & $\sim 1-4.8 \mathrm{~K}(\sim 14 \mathrm{GPa})$ & $P 3 m 1$ & Pnma (6.6 GPa) & $P 4 / n m m(19.1 \mathrm{GPa})$ \\
\hline $\mathrm{BiTeC}$ & $\mathrm{TI}$ & $\sim 4-8 \mathrm{~K}(\sim 11 \mathrm{GPa})$ & $\mathrm{P}_{3} m c$ & possible Pnma (5 GPa) & possible cubic (39 GPa) \\
\hline $\mathrm{Cd}_{3} \mathrm{As}_{2} 100$ & DSM & $2-4 \mathrm{~K}(8.5 \mathrm{GPa})$ & $I 4_{1} /$ acd & $P 2_{1} / c(3.5 \mathrm{GPa})$ & - \\
\hline $\mathrm{ZrTe}_{5}$ & DSM & $1.8-6 \mathrm{~K}(6.7 \mathrm{GPa})$ & $\mathrm{Cmcm}$ & $C 2 / m(6 \mathrm{GPa})$ & $P-1(21.2 \mathrm{GPa})$ \\
\hline $\mathrm{HfTe}_{5} 102$ & DSM & $1.8-4.8 \mathrm{~K}(5 \mathrm{GPa})$ & $\mathrm{Cmcm}$ & $C 2 / m(2.2 \mathrm{GPa})$ & $P-1(14.8 \mathrm{GPa})$ \\
\hline $\mathrm{TaP}^{103}$ & WSM & $1.8-3 \mathrm{~K}(70 \mathrm{GPa})$ & $I 4_{1} m d$ & $P-6 m 2(70 \mathrm{GPa})$ & - \\
\hline $\mathrm{MoTe}_{2} 104$ & WSM & $0.1-8.2 \mathrm{~K}$ (Ambient $\mathrm{P})$ & $P 2_{1} / m($ High $\mathrm{T})$ & $P m n 2_{1}$ (Ambient $\mathrm{P}$, low $\left.\mathrm{T}\right)$ & - \\
\hline $\mathrm{LaBi}$ & TSM & $4-8 \mathrm{~K}(3.5 \mathrm{GPa})$ & $F m-3 m$ & $P 4 / m m m(11 \mathrm{GPa})$ & - \\
\hline $\mathrm{WTe}_{2} 106 \mid 107$ & WSM & $\sim 3-7 \mathrm{~K}(2.5 \mathrm{GPa})$ & $\operatorname{Pmn} 2_{1}$ & - & - \\
\hline $\mathrm{MoF}^{108}$ & TPSM & $2.5-4 \mathrm{~K}(30 \mathrm{GPa})$ & $P-m 62$ & - & - \\
\hline $\mathrm{NbAs}_{2} 109$ & TSM & $2-2.6 \mathrm{~K}(12.8 \mathrm{GPa})$ & $C 2 / m$ & - & - \\
\hline
\end{tabular}

ilar to in $\mathrm{Bi}_{2} \mathrm{Te}_{3}{ }^{92} T_{c}$ can reach a maximum of $7 \mathrm{~K}$ below $50 \mathrm{GPa}$ when a body-centered cubic structure, similar to the $C 2 / m$ structure, appears at $28 \mathrm{GPa}{ }^{[4 \mid 112}$ The quasilinear temperature-dependent $H_{c 2}$ exceeds both the orbital and Pauli limits, which indicates an unconventional pairing state in $\mathrm{Bi}_{2} \mathrm{Se}_{3}$ under pressure ${ }^{95} \mathrm{Sb}_{2} \mathrm{Te}_{3}$ is another member of this TI family, and it becomes superconducting at $3 \mathrm{~K}$ when the applied pressure is increased up to $4 \mathrm{GPa} .{ }^{96}$ The $C 2 / m$ structure seems to be more stable above $12.9 \mathrm{GPa}$ in $\mathrm{Sb}_{2} \mathrm{Te}_{3}{ }^{\text {.97 }}$ According to DFT calculations using an experimental lattice at high pressure, the Dirac surface states remain at $6.9 \mathrm{GPa}$ in coexistence with superconductivity, which strongly suggests that this material is a promising TSC candidate.

Another TI material family, BiTeX $(\mathrm{X}=\mathrm{Cl}, \mathrm{Br}$, and I), consists of inversion-asymmetric TIs, which are usually called Rashba semiconductors because of the giant Rashba-type spin splitting!113]114 Robust Dirac surface states and large Rashba splitting have been revealed by ARPES $114\left[115 \mathrm{~A}\right.$ maximum $T_{c}$ of $5.2 \mathrm{~K}$ is reached at 23.5 GPa in BiTeI after two structural transitions. ${ }^{98}$ Interestingly, a nontrivial topology of the band structure is also obtained in the high pressure phase according to DFT calculations. In addition, similar pressure-induced superconducting phases have also been detected in $\mathrm{BiTeBr}^{98}$ and BiTeCl. ${ }^{99}$ Thus, members of the BiTeX family may be promising TSCs under pressure.

The properties of TIs under pressure are summarized in Table I. Fig.1(a) illustrates a sketched phase diagram of TIs under pressure, characterized by a structural transition before entering the superconducting phase. Only in some cases does the superconducting phase appear before the structural transition, for example, in $\mathrm{Bi}_{2} \mathrm{Te}_{3}{ }^{92}$ and $\mathrm{Sb}_{2} \mathrm{Te}_{3} \cdot{ }^{96}$ Therefore, determining whether there exist nontrivial topological states in the superconducting state requires further measurements, but the high pressure conditions make investigating topological superconductivity difficult.

\section{B. Topological semimetals under pressure}

The successful discovery of TIs has stimulated tremendous research on 3D DSMs with a 3D linear energy dispersion relation. Dirac nodes with fourfold degenerate crossings in the Brillouin zone have been confirmed in $\mathrm{Na}_{3} \mathrm{Bi},{ }^{116} \mathrm{Cd}_{3} \mathrm{As}_{2},{ }^{117} \mathrm{ZrTe}_{5},{ }^{118}$ etc. Intriguingly, a Dirac node will be split into two two-fold degenerate crossings with opposite chirality when a magnetic field is applied. These band crossings are named Weyl nodes $\frac{119}{}$ and exist in WSMs, where time reversal or inversion symmetries are broken because of magnetism or the noncentrosymmetric structure $\frac{120}{120}$ The Fermi arc formed by quasiparticle excitations of Weyl fermions was first confirmed in noncentrosymmetric transition-metal monophosphides (TaAs, ${ }^{\sqrt[121]{1}} \mathrm{TaP},{ }^{122} \mathrm{NbAs}, \sqrt{123} \mathrm{NbP}^{124}$ ). The negative magnetoresistance caused by chiral Weyl nodes when the electric field is parallel to the magnetic field is regarded as

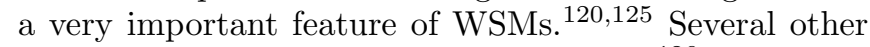
effects, such as the current jetting effect, $\frac{126}{126}$ could also cause a similar negative magnetoresistance ${ }^{127 / 128}$

Similar to TIs, DSMs and WSMs are also good platforms for exploring TSCs, and superconductivity can be induced by tuning the carrier density using pressure or doping. As expected, pressure-induced superconductivity is detected at $2 \mathrm{~K}$ in $\mathrm{Cd}_{3} \mathrm{As}_{2}$, a typical DSM, under 8.5 GPa $\frac{100}{}$ The superconductivity is quite robust and survives up to $50.9 \mathrm{GPa}$, with a maximal $T_{c}$ of $4.0 \mathrm{~K}$ under $21.3 \mathrm{GPa}$. Before the emergence of superconductivity in $\mathrm{Cd}_{3} \mathrm{As}_{2}$, the tetragonal $I 4_{1} /$ acd structure changes to a 
monoclinic $P 2_{1} / c$ phase at approximately $3.5 \mathrm{GPa}$. Superconductivity was observed at approximately $6 \mathrm{GPa}$ in another DSM, $\mathrm{ZrTe}_{5}$, where a structural transition from the ambient $C m \mathrm{~cm}$ phase to the monoclinic $C 2 / \mathrm{m}$ phase occurs 101 When the triclinic $P-1$ phase becomes stable at higher pressures above $21.2 \mathrm{GPa}$, a maximum $T_{c}$ of $\sim$ $6.0 \mathrm{~K}$ is obtained under $30 \mathrm{GPa}$. However, this structural transition, as supported by high-pressure X-ray diffraction measurements, may lead to the breakdown of the 3D DSM state in $\mathrm{Cd}_{3} \mathrm{As}_{2}{ }^{129}$ and $\mathrm{ZrTe}_{5}{ }^{101}$ The effect of such a structural transition on topological properties is still ambiguous. Some theoretical works suggest that this symmetry breaking during the structural transition may be favorable for the formation of a topological superconducting phase with surface Majorana fermions in $\mathrm{Cd}_{3} \mathrm{As}_{2}{ }^{130} \mathrm{HfTe}_{5}$ is an compound analogous to $\mathrm{ZrTe}_{5}$ in which two structural transitions occur, with one superconducting phase at $5 \mathrm{GPa}{ }^{102}$ First-principles calculations of these two high pressure phases showed the trivial properties in $\mathrm{HfTe}_{5}$ under pressure.

The search for TSCs and Majorana fermions in WSMs is also underway 131 through application of high pressure or the proximity effect between an s-wave superconductor and the surface states of a WSM ${ }^{132}$ When NbAs is subjected to pressure, no superconductivity exists up to 26 GPa while the ambient-pressure structure is retained! 133 However, if a higher pressure is applied to TaP, a superconducting transition is observed above $70 \mathrm{GPa}$, accompanied by a structural transition from $I 4_{1} m d$ to $P$ $6 m 2$ 103 Interestingly, superconductivity becomes more robust and even remains at $2 \mathrm{GPa}$ in pressurized $\mathrm{TaP}$ during the pressure decreasing process. DFT calculations indicate that a new WSM state appears in the $P$ $6 m 2$ phase, different from the ambient phase. This concurrence of superconductivity and the WSM state may result in a TSC for this WSM.

Type-II WSMs, such as $\mathrm{WTe}_{2}, \frac{134}{\mathrm{MoTe}_{2}, 135}$ $\mathrm{Mo}_{x} \mathrm{~W}_{1-x} \mathrm{Te}_{2}, \frac{136}{}$ and $\mathrm{Ta}_{3} \mathrm{~S}_{2}$, ${ }^{137}$ have drawn increasing attention due to the tilted and protected Weyl-type band crossings. ${ }^{138}$ Exploring TSCs among type-II WSMs is also attractive because they are more common than type-I WSMs. In the type-II WSM WTe 2 , an extremely large magnetoresistance (XMR) was reported due to its electron-hole compensation! ${ }^{[39}$ With increasing pressure, superconductivity is induced by suppression of XMR in $\mathrm{WTe}_{2}$, 106/107 as shown in Fig 1(b). The sign of the Hall coefficients changes without a structural transition when superconductivity emerges in the vicinity of 10.5 GPa. Thus, reasonably, the mismatch between the electron and hole carrier densities at high pressure could contribute to the suppression of XMR!106 The explanation of the occurrence of superconductivity in $\mathrm{WTe}_{2}$ under pressure is related to the increase of the density of states.107 Another type-II WSM, MoTe 2 , shows a superconducting transition at $0.1 \mathrm{~K}$ even under ambient pressure 104 After a structural transition at $240 \mathrm{~K}$ from the $\mathrm{T}^{\prime}$ phase to the $\mathrm{T}_{d}$ phase, WSM states could be observed, as expected, in the $\mathrm{T}_{d}$ phase by
ARPES 140141 In addition, a high pressure will increase $T_{c}$ to a maximum value of $8.2 \mathrm{~K}$, and the structural transition temperature will decrease with increasing pressure. 104

Moreover, superconductivity under pressure has also been detected in several other topological semimetals, such as LaBi, ${ }^{105 \mid 142}$ MoP, ${ }^{108 \mid 143}$ and $\mathrm{NbAs}_{2}$. 109|144 The high-pressure-induced superconductivity in these topological materials is seemingly accompanied by crystal structure symmetry breaking, as seen in Fig 1(b) and Table[I] which usually changes the topological features. However, superconductivity and nontrivial topological properties should simultaneously exist in a potential TSC. High pressure conditions make performing crucial experiments, such as ARPES, to test the nontrivial topological properties difficult, and pressure-induced structural transitions make such experiments even more complicated. Consequently, investigating the topological semimetals that become superconducting without a structural transition under pressure, such as $\mathrm{WTe}_{2}, \frac{106 \mid 107}{10}$ $\mathrm{MoP}, 108 \mid 143$ and $\mathrm{NbAs}_{2}, \frac{109] 144}{15}$ is relatively more meaningful. For example, pressure-induced superconductivity is obtained at $2.63 \mathrm{~K}$ and $12.8 \mathrm{GPa}$ in the topological semimetal $\mathrm{NbAs}_{2}$ without a structural transition.109 A linear Dirac dispersion was recently detected in $\mathrm{NbAs}_{2}$ through magneto-optical measurements.144 A negative magnetoresistance is widely observed in $\mathrm{NbAs}_{2}$, 1451146 as predicted ${ }^{147}$ In addition, as a semimetal with triply degenerate nodal points, ${ }^{143} \mathrm{MoP}$ exhibits superconductivity when subjected to pressure above $30 \mathrm{GPa}$, while no structural transition occurs up to $60 \mathrm{GPa}+108$ DFT calculations with SOC uncovered the coexistence of Weyl points and triply degenerate nodal points under high pressure. In these systems, topological properties and superconductivity coexist under pressure, which may be a new platform to explore TSC candidates.

\section{TIP-INDUCED SUPERCONDUCTORS}

Tip-induced superconductivity in topological semimetals has been recently observed in hard point contact experiments.148 Usually, hard point contact measurement is a useful method to study the gap structure of superconductors. This technique has become a new method for tuning nonsuperconducting materials into superconducting states, similar to other modulation methods, such as electric field gating, high pressure application, and chemical doping. Obviously, this technique has the advantage that the point contact spectroscopy (PCS) can be simultaneously measured when the sample is tuned. Thus, hard point contact measurement of topological semimetals offers a new way to trigger and detect topological superconductivity.

When the hard tip method is implemented on the DSM $\mathrm{Cd}_{3} \mathrm{As}_{2}$, the resistance begins to drop at $3.9 \mathrm{~K}$, suggesting a superconducting transition.149 Another group reported a similar experiment in which $\mathrm{Cd}_{3} \mathrm{As}_{2}$ shows $T_{c}$ 
TABLE II. Summary of doping-induced superconductivity in topological insulators (TIs) or topological crystalline insulators (TCIs). $T_{c}^{\max }$ is the maximum value of the superconducting transition temperature for various doping contents $x$.

\begin{tabular}{|c|c|c|c|}
\hline Material & Type & $T_{c}^{\max }$ & $x$ \\
\hline $\mathrm{Cu}_{x} \mathrm{Bi}_{2} \mathrm{Se}_{3} 110$ & $\mathrm{TI}$ & $3.8 \mathrm{~K}$ & $0.09<x<0.64$ \\
\hline $\mathrm{Sr}_{x} \mathrm{Bi}_{2} \mathrm{Se}_{3}$ & $\mathrm{TI}$ & $2.9 \mathrm{~K}$ & $0.058<x<0.1$ \\
\hline $\mathrm{Nb}_{x} \mathrm{Bi}_{2} \mathrm{Se}_{3} 158$ & $\mathrm{TI}$ & $3.2 \mathrm{~K}$ & 0.25 \\
\hline $\mathrm{Tl}_{x} \mathrm{Bi}_{2} \mathrm{Se}_{3} 159 \mid 160$ & $\mathrm{TI}$ & $2.28 \mathrm{~K}$ & 0.6 \\
\hline $\mathrm{Cu}_{x}(\mathrm{PbSe})_{5}\left(\mathrm{Bi}_{2} \mathrm{Se}_{3}\right)_{6} 161$ & TI & $2.85 \mathrm{~K}$ & $0.3<x<2.3$ \\
\hline $\mathrm{Sn}_{1-x} \operatorname{In}_{x} \mathrm{Te}^{162}$ & TCI & $4.7 \mathrm{~K}$ & $0.017<x<0.4$ \\
\hline$\left(\mathrm{Pb}_{0.5} \mathrm{Sn}_{0.5}\right)_{1-x} \operatorname{In}_{x} \mathrm{Te}^{164}$ & TCI & $4.7 \mathrm{~K}$ & $0.1<x<0.3$ \\
\hline
\end{tabular}

$=5.8 \mathrm{~K} ! 150$ This difference in $T_{c}$ may originate from the pressure applied by the hard tip, and the actual pressure on the sample is difficult to measure in such experiments. Charge transfer may also exist between the tip and the sample, which would lead to the charge doping effect, similar to the chemical doping case. When a magnetic field is employed perpendicular to the plane on which the pressure is applied, the magnetic-field-dependent $T_{c}$ shows a concave curvature in the low magnetic field regime, indicating unconventional superconductivity 150 In the PCS results of the above two reports, superconducting gaps are successfully observed with ZBPs at zero energy. The ZBP behaviors at various temperatures and magnetic fields suggest the existence of MZMs in $\mathrm{Cd}_{3} \mathrm{As}_{2}$. A tip-induced superconducting transition, with $T_{c}=5.9 \mathrm{~K}$, and ZBPs have also been observed in the WSM TaAs $\frac{148}{14}$ However, further theoretical and experimental studies are highly desired to understand the exact mechanisms that occur in the tip contact region and how to precisely analyze PCS results.

\section{DOPED TOPOLOGICAL MATERIALS}

Chemical doping, including doping and intercalation, is widely used to induce superconductivity in electron correlation systems and to study interesting physics-like phase transitions by modulating the carrier density density/Fermi level or introducing chemical pressure. For example, superconductivity was realized in fluorine-doped LaOFeAs through electron doping, thus beginning the era of iron-based superconductors ${ }^{151}$ Chemical pressure can also be induced by doping with elements with smaller radii, such as $\mathrm{P}$-for-As doping in $\mathrm{BaFe}_{2} \mathrm{As}_{2}{ }^{[152]}$ In addition, some fantastic physical phenomena are observed in magnetic-element-doped systems, such as the quantum anomalous Hall effect in a chromium-doped TI, where the time reversal symmetry is broken,, 153 and dilute ferromagnetism in dilute magnetic semiconductors and dilute magnetic oxides $\frac{154}{154}$ Therefore, doping or intercalation of a TI is naturally an interesting approach to exploring potential TSCs. $\frac{155}{}$

\section{A. Doping of topological insulators}

$\mathrm{Bi}_{2} \mathrm{Se}_{3}$ is one of the most studied TIs. ${ }^{89}$ Copper elements can be intercalated in the van der Waals gaps between $\mathrm{Bi}_{2} \mathrm{Se}_{3}$ layers to obtain superconductivity, with $T_{c}=3.8 \mathrm{~K}$, in $\mathrm{Cu}_{x} \mathrm{Bi}_{2} \mathrm{Se}_{3}$, and the content of intercalated $\mathrm{Cu}$ is $0.12 \leq x \leq 0.15$ in the samples prepared by the solid phase reaction. ${ }^{110}$ The content of $\mathrm{Cu}$ in $\mathrm{Cu}_{x} \mathrm{Bi}_{2} \mathrm{Se}_{3}$ synthesized by the electrochemical intercalation technique can be better controlled, and the superconducting shielding fractions are as large as $\sim 50 \% \frac{156}{15}$ These interesting structures and physics immediately drew extensive attention. A full energy gap in the bulk superconductivity was observed by temperature-dependent specific heat 165 and STM measurements ${ }^{166}$ More intriguingly, ARPES measurements revealed the existence of nontrivial surface states ${ }^{167}$ The upper critical field data measured under various pressures are not consistent with the behavior of a weak coupling, orbital-limited, spin-singlet superconductor, suggesting a spin-triplet or an anisotropic spin-singlet state in $\mathrm{Cu}_{0.3} \mathrm{Bi}_{2} \mathrm{Se}_{3} \cdot{ }^{168}$ Moreover, an evident ZBP caused by Andreev bound states consisting of Majorana fermions was obtained with the 'soft' point contact technique ${ }^{[169}$ All of these experiments indicate that $\mathrm{Cu}_{x} \mathrm{Bi}_{2} \mathrm{Se}_{3}$ is a good candidate for time-reversalinvariant TSCs with massless Majorana fermions in the surface states 12

More interestingly, nematic superconductivity has been observed in $\mathrm{Cu}_{x} \mathrm{Bi}_{2} \mathrm{Se}_{3}$, where the spin rotation symmetry is broken below the superconducting transition temperature. ${ }^{[70 \mid 171}$ When an external magnetic field is applied to the hexagonal plane, two-fold symmetry of the Knight shift is detected by ${ }^{77} \mathrm{Se}$ NMR measurements below $T_{c}=3.4 \mathrm{~K}$ in $\mathrm{Cu}_{0.3} \mathrm{Bi}_{2} \mathrm{Se}_{3}$, which possesses a three-fold rotational symmetry lattice $\frac{170}{}$ This symmetry breaking behavior suggests a pseudo-spin-triplet state of Cooper pairs. Further measurements, such as specific heat, upper critical field ${ }^{171}$ and STM measurements, ${ }^{172}$ confirmed this spontaneous rotational symmetry breaking (RSB) superconductivity, which was named as a new class of nematic superconductivity with odd-parity pairing ${ }^{171 \mid 173}$ Nematic superconductors in which spontaneous RSB exists in the amplitude factor of the superconducting gap are completely different from superconductors hosting spontaneous RSB in the phase factor of the superconducting gap, which can be observed by phase-sensitive junction techniques. ${ }^{26}$

Bulk superconductivity with a large superconducting volume fraction $(\sim 91.5 \%)$ has also been detected in $\mathrm{Sr}_{x} \mathrm{Bi}_{2} \mathrm{Se}_{3}$, which exhibits a maximum $T_{c}=2.5$ $\mathrm{K}$ when $x \sim 0.06, \frac{157}{10}$ and a higher $T_{c}$ of $2.9 \mathrm{~K}$ was found in an $x=0.1$ sample ${ }^{1111}$ Instead of $\mathrm{Cu}$ and $\mathrm{Sr}$ intercalation, niobium intercalation in $\mathrm{Bi}_{2} \mathrm{Se}_{3}$ can enhance $T_{c}$ to $3.2 \mathrm{~K}$, with a $100 \%$ superconducting volume fraction ${ }^{158}$ The intercalation of $\mathrm{Cu}$ in the interesting layer-structured $\mathrm{TI}(\mathrm{PbSe})_{5}\left(\mathrm{Bi}_{2} \mathrm{Se}_{3}\right)_{6}$, which is viewed as a heterostructure with PbSe units and $\mathrm{Bi}_{2} \mathrm{Se}_{3}$ units,$\frac{174}{}$ results in superconductivity with a maximum 
$T_{c}=2.85 \mathrm{~K}{ }^{161}$ Unconventional superconductivity with a nodal gap in $\mathrm{Cu}_{x}(\mathrm{PbSe})_{5}\left(\mathrm{Bi}_{2} \mathrm{Se}_{3}\right)_{6}$ was verified by specific heat $\frac{161}{1}$ and ARPES measurements, $\frac{175}{17}$ and the topological state and superconductivity with a nodal gap may be able to coexist! 14 Similar to $\mathrm{Cu}_{x} \mathrm{Bi}_{2} \mathrm{Se}_{3}$, nematic order with two-fold symmetry was also revealed

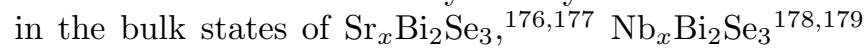
and $\mathrm{Cu}_{x}(\mathrm{PbSe})_{5}\left(\mathrm{Bi}_{2} \mathrm{Se}_{3}\right)_{6}$. $\frac{180}{1}$ Therefore, these intercalated systems offer a good platform to study this new type of TSC, and various theories also support this point of view! $12|181| 182$

\section{B. Doping of topological crystalline insulators}

Unlike TIs, TCIs exhibit topological properties that originate from crystal symmetries when SOC is not taken into consideration ${ }^{[183]}$ For example, gapless surface states in the TCI SnTe with the Fm-3m space group are protected by the mirror symmetry of the crystal with respect to the $\{110\}$ mirror plane. A gap in the surface states will open if the mirror symmetry is broken by an in-plane magnetic field ${ }^{184}$ Subsequently, ARPES measurements revealed a metallic Dirac-cone surface state in SnTe ${ }^{\sqrt{185}}$ which was successfully confirmed as a TCI. When indium is doped into SnTe, superconductivity is observed in $\operatorname{Sn}_{1-x} \operatorname{In}_{x}$ Te for $x>1.7 \%$, while the ferroelectric structural phase transition (at $95 \mathrm{~K}$ in $\mathrm{SnTe}$ ) is completely suppressed for $x>4 \% ! \frac{162}{\square} T_{c}$ is enhanced to $4.7 \mathrm{~K}$ at $x=0.4$, and the Ginzburg-Landau parameter $\kappa=56.4(8)$ estimated from the upper and lower critical fields suggests a type-II superconductor 163 A full superconducting gap in $\mathrm{Sn}_{1-x} \mathrm{In}_{x}$ Te has been established by thermal conductivity $\frac{186]}{18}$ specific heat $\frac{187}{18}$ and $\mu \mathrm{SR}$ measurements ${ }^{188}$ Intriguingly, PCS experiments on a $x=0.045$ sample uncovered a large magnitude zerobias conductance peak, which was caused by the surface Andreev bound states $\frac{189}{18}$ High-resolution ARPES measurements indicate that the topological surface state is retained in $\mathrm{Sn}_{1-x} \mathrm{In}_{x} \mathrm{Te}{ }^{190}$ All of these data provide evidence for topological superconductivity in $\mathrm{Sn}_{1-x} \mathrm{In}_{x} \mathrm{Te}$.

Because of the hole-doped nature of $\mathrm{SnTe}, \mathrm{Pb}$ doping can tune SnTe or SnSe to either n-type or p-type conductivity, and a large doping range may induce topological phase transitions $184191[193$ A topological phase transition indeed occurs in $\mathrm{Pb}_{1-x} \mathrm{Sn}_{x}$ Te at $x=0.25$ from a trivial band insulator phase $(\mathrm{PbTe})$ to a TCI phase (SnTe) ${ }^{184|191| 192}$ The analogous compound $\mathrm{Pb}_{1-x} \mathrm{Sn}_{x} \mathrm{Se}$ also possesses a TCI state at $x=0.23, \frac{193}{193}$ and further transport experiments revealed its massive Dirac bulk states ${ }^{194}$ Moreover, interestingly, the indium substituted compound $\left(\mathrm{Pb}_{0.5} \mathrm{Sn}_{0.5}\right)_{1-x} \operatorname{In}_{x} \mathrm{Te}$ displays superconductivity in the range of $0.1<x<0.3$, with maximum $T_{c}=$ $4.7 \mathrm{~K} ! \frac{164}{16}$ The superconducting state is fully gapped without any in-gap states based on STM measurements ${ }^{195}$

Compared with the high pressure technique, the doping method can not only induce superconductivity in TIs or TCIs but also allow further experiments to con- firm topological superconductivity with odd parity, such as PCS experiments to observe the ZBP,169|189 specific heat $\frac{171]}{S^{17} M^{172}}$ or NMR ${ }^{[170}$ measurements to verify spontaneous RSB/nematicity, and ARPES measurements to detect gapless surface states. $\frac{167 / 190}{\text { The doped }}$ TIs and TCIs hosting superconductivity are summarized in Table II. A sketched phase diagram vs. doping concentration is plotted in Fig 1(a), and topological phase transitions from a TI/TCI to a TSC are well tunable by doping. Therefore, $\mathrm{Cu}_{x} \mathrm{Bi}_{2} \mathrm{Se}_{3}$ and $\mathrm{Sn}_{1-x} \mathrm{In}_{x}$ Te systems are promising TSCs 14

\section{ARTIFICIAL STRUCTURES}

\section{A. Gate-induced TSCs}

Field-effect transistors (FETs) are the core of the gating technique, which can help induce superconductivity by modulating the carrier density of $2 \mathrm{D}$ materials. 196 Three types of gating methods are well developed, including those based on metal-insulator-semiconductor (MIS) FETs, ${ }^{196}$ electric double layer (EDL) FETs ${ }^{197 / 198}$ and solid ion conductor (SIC) FETs ${ }^{199}$ The limits of the carrier density in a $2 \mathrm{D}$ system modulated by the first two types are $2 \times 10^{13} \mathrm{~cm}^{-2[196}$ and $1 \times 10^{15} \mathrm{~cm}^{-2} \stackrel{203}{2}$ The SIC FET can contain $\mathrm{Li}^{+}$in the $2 \mathrm{D}$ material, and the amount of intercalated $\mathrm{Li}^{+}$can be comparable to the quantity of the base compound elements ${ }^{[199}$ Therefore, these gating techniques can be used to study complex phase diagrams of $2 \mathrm{D}$ materials by controlling their carrier density.

With a gapless, massless and chiral Dirac spectrum,204205 graphene can be tuned to a superconducting state when a 'magic' angle exists in twisted bilayer graphene. ${ }^{206} T_{c}=1.7 \mathrm{~K}$ is observed when the 'magic' angle is $1.1^{\circ}$. Through the tunability of the carrier density, the superconducting diagram exhibits similar characteristics to that of cuprates. Flat bands emerge in the band structure of twisted bilayer graphene, and a Mott-like insulator phase appears when the Fermi level is tuned at the half-filling of these flat bands, while two superconducting domes appear adjacent to this half-filling state ${ }^{206 / 207}$ These experiments offer new insights into unconventional superconductors and quantum spin liquids. Moreover, theoretic predictions have been performed and indicate possible topological superconductivity in twisted multilayer graphene, 208 which can host spin triplet $d+i d$ order and topologically protected gapless edge states. Therefore, topological superconductivity in this system is worthy of further investigation.

Much progress has also been made in the FET experiments of topological semimetals. When the 3D typeII WSM $\mathrm{WTe}_{2}$ is fabricated into a monolayer crystal, the hallmark transport conductance with approximately $e^{2} / h$ per edge is observed up to $100 \mathrm{~K}$, indicating a quantum spin Hall state in monolayer $\mathrm{WTe}_{2}{ }^{[209}$ If the carrier density in monolayer $\mathrm{WTe}_{2}$ is tuned by moderat- 
ing electrostatic gating, then a quantum phase transition emerges with increasing gate voltage, and the quantum spin Hall state can transform into a superconducting state.210211 The maximum $T_{c}$ is $\sim 1 \mathrm{~K}$ when the $2 \mathrm{D}$ carrier density is modulated to be more than $1 \times 10^{13} \mathrm{~cm}^{-2}$. The in-plane upper critical field is much higher than the Pauli limit value. Such high $\mathrm{H}_{c 2}$ could originate from various phenomena, such as Ising-type superconductivity, 197 a reduced electron $g$-factor, a spin-triplet pairing state, or strong spin-orbit scattering. ${ }^{210} 212$ The suppression of the helical edge states by the magnetic field through breaking of the time reversal symmetry seems to persist in the superconducting state, which implies nontrivial properties in monolayer $\mathrm{WTe}_{2}, 211$ although the pairing state requires more evidence.

Among these three FET methods, the MIS FET technique is the cleanest one, and only an external electronic field is applied to tune the carrier density, in contrast with EDL FET and SIC FET techniques. Therefore, in the next subsection on heterostructures or Josephson junctions, the MIS FET technique is widely used to control the Fermi level of heterostructures and thus modulate the superconducting state!7|213 Therefore, gating techniques are very attractive for exploring TSCs among topological materials.

\section{B. Nanowire/superconductor heterostructures}

One effective approach to obtaining TSCs with Majorana fermions in artificial devices is placing semiconductor nanowires on an s-wave superconductor. Many similar proposals to detect MZMs in this type of heterostructure have been made! $7|13| 214|216|$ The general experimental setup is as follows. First, the $1 \mathrm{D}$ semiconductor nanowire should exhibit large Rashba SOC, which can be regarded as a spin-orbital effective field $\mathbf{B}_{\text {so }} \propto \mathbf{p} \times \mathbf{E}$ (where $\mathbf{E}$ is the electric field vertical to the nanowire, while $\mathbf{p}$ is the momentum along the nanowire). This $\mathbf{B}_{\text {so }}$ is perpendicular to the nanowire and splits the parabolic band, which results in crossing of two spin-orbital bands at $p=0$ (the red band and blue band), as shown in Fig. 2 . Then, if an external magnetic field $B$ is applied along the axis of the nanowire, a Zeeman gap $2 E_{Z}=g \mu_{B} B$ opens at this crossing point, where $g$ is the Landé $g$-factor and $\mu_{B}$ is the Bohr magneton. The proximity effect of the s-wave superconductor and the nanowire will change the band structure by inducing a superconducting gap $\Delta_{\text {ind }}$ at $p=p_{F}$ in the nanowire, marked by the yellow arrows. The s-wave superconductor can induce fully gapped superconductivity in the nanowire through the proximity effect. Then, the Zeeman gap at $p=0$ can be modulated to control the topological phase of the nanowire by changing the external magnetic field $B$. A topological phase transition emerges at $E_{Z}=\sqrt{\Delta_{i n d}^{2}+\mu^{2}}$, where $\mu$ is the chemical potential, as seen in Fig.2. When $E_{Z}$ is less than $\sqrt{\Delta_{\text {ind }}^{2}+\mu^{2}}$, the nanowire is actually a trivial superconductor similar to the proximate s-wave super- conductor. Subsequently, with increasing $E_{Z}$, the gap at $p=0$ will be reduced to zero, and zero-energy bound states formed by Majorana states will appear at each end of the nanowire. Therefore, if $E_{Z}>\sqrt{\Delta_{i n d}^{2}+\mu^{2}}$, the nanowire enters into a topological superconducting state. $13 \mid 217$

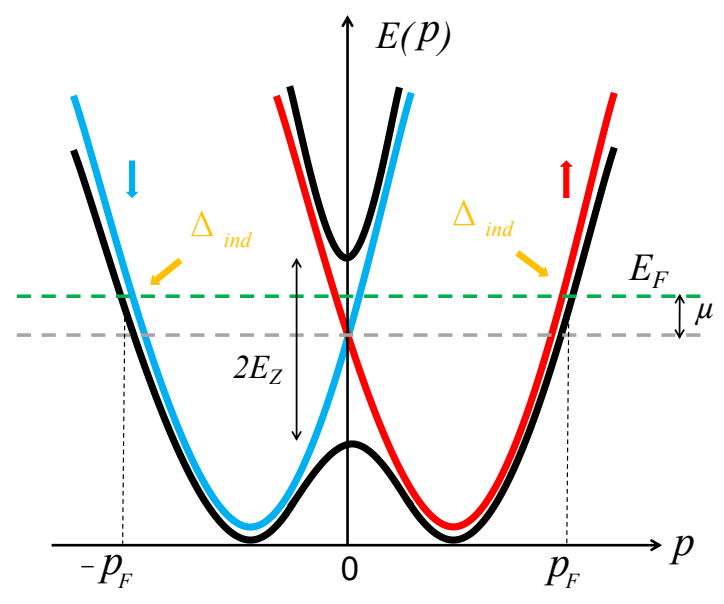

FIG. 2. The band evolution of a 1D nanowire coupled to an s-wave superconductor. First, the degenerate parabolic band of the 1D nanowire can split due to Rashba SOC. Then, the external magnetic field along the nanowire causes a Zeeman gap at the crossing point $p=0$. Moreover, a superconducting gap $\Delta_{i n d}$ is introduced in the nanowire at $p=p_{F}$ by the proximity effect of the s-wave superconductor, which is not shown in the figure. A different band structure can be obtained by tuning the value of the Zeeman energy $E_{Z} \cdot \frac{217}{}$

Following the above idea, Majorana bound states have been successfully observed in the heterostructure consisting of normal state (gold)-InSb-superconductor (NbTiN) 19 Semiconductor InSb nanowires possess strong SOC and a large $g$-factor $(g \approx 50)$. These characteristics greatly contribute to modulating the Zeeman gap $2 E_{Z}$, which must be larger than the induced superconducting gap. The induced superconducting gap here is $\Delta_{\text {ind }} \approx 0.25 \mathrm{meV}$, and the Zeeman gap $E_{Z} / B \approx$ $1.5 \mathrm{meV} / \mathrm{T}$. Therefore, when $B>0.15 \mathrm{~T}$, a topological phase transition will occur because $E_{Z}$ begins to exceed $\Delta_{\text {ind }}$. Majorana bound states have been experimentally observed at the boundary of the nanowire at $B=0.25 \mathrm{~T}$. By varying the gate voltage, the chemical potential $\mu$ can be controlled to lower the critical $B$ at which the topological phase transition emerges. In addition, with increasing angle between the nanowire and external magnetic field $B$, the ZBP originating from the Majorana fermion will finally disappear in the perpendicular case. The amplitude of $d I / d V$ at the ZBP usually deviates from the theoretical value of $2 e^{2} / h$ at zero temperature ${ }^{218 \mid 219}$ due to the finite measurement temperature, where $h$ is the Planck constant and $e$ is the electric charge.

The observation of MZMs has also been reported in a 
similar device with an Al-InAs heterostructure.217 InAs is a semiconductor with large SOC and $g \approx 20$. A small external magnetic field in this device is required to observe the ZBPs because the induced superconducting gap $\Delta_{i n d} \approx 0.05 \mathrm{meV}$ is very small, which is related to the magnitude of the superconducting gap of the s-wave superconductor aluminum. In addition, the signature of MZMs can be detected in several other devices, such as InSb-NbTiN, $\stackrel{220}{ } \mathrm{InSb}_{\mathrm{Nb}} \stackrel{221}{ }$ and InAs-Al heterostructures. 222

To avoid other effects, such as the Kondo effect ${ }^{223}$ or the so-called ' 0.7 anomaly', 224225 which could also cause ZBPs in the conductivity measurements of ferromagnetic atomic chains on an s-wave superconductor, a radiofrequency/microwave technique was used for a.c. Josephson junctions (a $\mathrm{Nb} / \mathrm{InSb} / \mathrm{Nb}$ device) to detect edge states. ${ }^{226}$ The conventional supercurrent is carried by charge $2 e$ Cooper pairs, with a height of the quantized voltage steps (Shapiro steps) of $\Delta V=h f_{0} / 2 e$ in the $I-V$ curves, where $f_{0}$ is the frequency of microwave excitation. When a high magnetic field is applied, the height of the quantized voltage steps changes to $h f_{0} / e$, indicating the appearance of charge $e$ quasiparticles formed by two Majorana fermions in the supercurrent. Therefore, this doubling of the Shapiro steps is a characteristic signature of MZMs.

\section{TI/superconductor heterostructures}

The proximity effect in TI/superconductor heterostructures has also been employed as an effective method to induce MZMs ${ }^{10}$ Instead of a semiconductor nanowire/superconductor heterostructure, a device with a linear junction between two superconductors mediated by a TI was fabricated, and a 1D wire of Majorana fermions was engineered along the width of this junction. Various experiments have been performed to obtain evidence of Majorana fermions. $227+234$ However, detecting the feature of Majorana fermions is quite difficult. A very important experiment for this type of device is the observation of the Fraunhofer-type dependence of the critical current $I_{c}$ modulated by the magnetic field! 2281229

$$
I_{c}(B)=I_{c}(0)\left|\sin \left(\frac{\pi \Phi_{J}}{\Phi_{0}}\right) /\left(\frac{\pi \Phi_{J}}{\Phi_{0}}\right)\right|,
$$

where $\Phi_{J}$ is the flux through the effective junction area and $I_{c}(0)$ is the superconducting critical current at zero magnetic field. The usual $2 \pi$-periodic Josephson supercurrent oscillates with a periodicity of $\Delta B=\Phi_{0} / A$ ( $A$ is the area of the junction). However, a $4 \pi$-periodic Josephson supercurrent emerges $\left(\Delta B=2 \Phi_{0} / A\right)$, suggesting the existence of MZMs 233

This $4 \pi$-periodic Josephson effect was successfully obtained in the device fabricated with strained and undoped HgTe on a CdTe substrate, in which superconductor $\mathrm{Nb}$ electrodes were used!22 The Shapiro steps $V_{n}=n h f / 2 e$ are observed in the $I-V$ curves under high radiofrequency. The length of the current steps in the Shapiro steps presents a $4 \pi$-periodic behavior, which may be related to a p-wave superconductor due to a doublet of topologically protected gapless Andreev bound states. In a similar device of $\mathrm{HgTe} / \mathrm{HgCdTe}$ quantum wells, helical edge states appear in the supercurrent distributions upon controlling the width of the device, and this quantum spin Hall effect provides another insight into TSCs.24 Subsequently, another type of HgTe-based Josephson junction was fabricated to measure the radiofrequency emission spectra. The half Josephson frequency originating from the $4 \pi$-periodic gapless Andreev doublet around zero energy was confirmed in this device, although the coherence time of $0.3-4$ ns was very short.23

In another experiment, a superconductor was coupled to a thin film of a quantum anomalous Hall insulator to detect the chiral Majorana fermion modes, i.e., a superconductor $\mathrm{Nb}$ bar was fabricated on a $\left(\mathrm{Cr}_{0.12} \mathrm{Bi}_{0.26} \mathrm{Sb}_{0.62}\right)_{2} \mathrm{Te}_{3}$ film. ${ }^{235}$ An external magnetic field was applied to modulate the topological state of the device, and a half-integer plateau of the longitudinal conductance $\left(e^{2} / 2 h\right)$ was obtained. This unique transport signature indicates the existence of MZMs. Therefore, this type of device may become a promising component in topological quantum computation.

ARPES and STM are both powerful techniques to detect the MZMs in TI films grown on superconductors. ${ }^{7 / 20 \mid 236}$ A straightforward approach is to grow $\mathrm{Bi}_{2} \mathrm{Se}_{3}$ thin films by molecular beam epitaxy (MBE) on the s-wave superconductor $\mathrm{NbSe}_{2}$. Experimentally, a superconducting gap is observed in the $\mathrm{Bi}_{2} \mathrm{Se}_{3}$ film, coexisting with topological surface states, which may generate topological superconductivity in the TI film.237 When $\mathrm{Bi}_{2} \mathrm{Se}_{3}$ films are grown on the d-wave superconductor $\mathrm{Bi}_{2} \mathrm{Sr}_{2} \mathrm{CaCu}_{2} \mathrm{O}_{8+\delta}$, fully gapped topological surface states are detected by ARPES.236 This isotropic superconducting gap structure is different from the bulk superconducting gap structure and may provide another route to realizing MZMs. Moreover, MZMs located in the vortex core in the $\mathrm{Bi}_{2} \mathrm{Te}_{3} / \mathrm{NbSe}_{2}$ heterostructure have been successfully observed by STM measurements.20|21 To conclude, TI/superconductor heterostructures also offer a good device platform to realize realistic topological quantum computation.

\section{Natural heterostructures}

In addition to artificial heterostructures, some attempts have been made to synthesize natural heterostructures to realize TSCs. For such natural heterostructures, the samples are usually bulk single crystals. A good example of natural heterostructures whose TI features have been experimentally confirmed is $\left(\mathrm{PbSe}_{5}\left(\mathrm{Bi}_{2} \mathrm{Se}_{3}\right)_{3 m}(m\right.$ $=1,2, \ldots){ }^{174}$ As mentioned in the previous section, this compound can be viewed as the alternation of $(\mathrm{PbSe})_{5}$ layer units and $\left(\mathrm{Bi}_{2} \mathrm{Se}_{3}\right)_{3}$ layer units. $\mathrm{Bi}_{2} \mathrm{Se}_{3}$ is a TI, and $\mathrm{PbSe}$ is an ordinary insulator. The inversion sym- 
metry breaking at the interface of these two units will induce Rashba splitting of the topological surface state at $m=2$. With increasing $m$, a topological phase transition occurs from a trivial phase at $m=1$ to a TI phase at $m \geq 2$. When $\mathrm{Cu}$ is intercalated in the $\mathrm{Bi}_{2} \mathrm{Se}_{3}$ units of this compound, forming $\mathrm{Cu}_{x}(\mathrm{PbSe})_{5}\left(\mathrm{Bi}_{2} \mathrm{Se}_{3}\right)_{6}$, a superconducting transition emerges when the value $x$ is between 0.3 and 2.5 161 Unconventional superconductivity with a nodal gap and nematic superconductivity have also been observed, as mentioned in subsection A of section IV. Therefore, $\mathrm{Cu}_{x}(\mathrm{PbSe})_{5}\left(\mathrm{Bi}_{2} \mathrm{Se}_{3}\right)_{6}$ may be a candidate for TSCs with a nodal gap 14 Although superconductivity exists in the Ag-doped $(\mathrm{PbSe})_{5}\left(\mathrm{Bi}_{2} \mathrm{Se}_{3}\right)_{3}(m=1)$ samples, with $T_{c}=1.7 \mathrm{~K}, 238$ no superconducting transition appears in the Ag-doped TI samples $(\mathrm{PbSe})_{5}\left(\mathrm{Bi}_{2} \mathrm{Se}_{3}\right)_{6}$ $(m=2)$.

Other natural structures, such as misfit layer compounds, also exist. ${ }^{239}$ Misfit compounds are a large family characterized by $(\mathrm{MX})_{1+y}\left(\mathrm{TX}_{2}\right)_{n}(y=0.08-0.28, n=1$, $2,3)$, where $\mathrm{M}=\mathrm{Sn}, \mathrm{Pb}, \mathrm{Sb}, \mathrm{Bi}$ or a lanthanide; $\mathrm{X}=\mathrm{S}$, Se or Te; and $\mathrm{T}=\mathrm{Ti}, \mathrm{V}, \mathrm{Cr}, \mathrm{Nb}$ or $\mathrm{Ta}$. Among these compounds, the $\mathrm{TX}_{2}$ layer is usually a superconducting unit, and the MX layer can be regarded as a modulated structural unit. This interesting natural heterostructure could possess an abnormally large upper critical field, 240 which may originate from multiband effects or similar spin-valley locking in the $2 \mathrm{D}$ limit case 197 The misfit compounds exhibit inversion symmetry breaking at the interface of the heterostructure, which may be accompanied by the Rashba effect in a strong SOC system, such as $(\mathrm{PbSe})_{5}\left(\mathrm{Bi}_{2} \mathrm{Se}_{3}\right)_{6}$. These natural heterostructures lower the dimensionality of crystals, which could contribute to a large anisotropic upper critical field, such as in the $2 \mathrm{D}$ limit case of ion-gated $\mathrm{MoS}_{2}{ }^{241}$ and $\mathrm{NbSe}_{2}{ }^{242}$ bilayers. If the MX layer is composed of topological materials, such as the predicted monolayer TCIs SnTe, PbS, PbSe, and $\mathrm{PbTe}, \frac{184|243| 246}{24}$ the proximity effect of the superconducting $\mathrm{TX}_{2}$ layer may induce Majorana bound states ${ }^{10}$ in the adjacent surface states of the TI layers, similar to those in the artificial TI/superconductor heterostructures mentioned in the previous subsection. Unfortunately, no experiments confirming the topological features in these misfit compounds have been reported.

\section{E. Other artificial structures}

Moreover, several other heterostructures to realize MZMs have been studied. The heterostructure of ferromagnetic atomic chains on an s-wave superconductor has been successfully fabricated, and ZBPs can be observed in these devices, which may be caused by the Kondo effect or disorder. $220 \mid 247+250$ ZBPs at the boundary of the ferromagnetic atomic chain can be successfully detected in the artificial structure of magnetic atomic chains on the surface of an s-wave superconductor by means of a high-resolution spectroscopic imaging technique.251

In other artificial structures, when quantum Hall edges are coupled with a superconductor, new excitations, such as non-Abelian anyons, will emerge, and nonlocal transport could possibly be used to detect Majorana fermions.252 Subsequently, graphene/superconductor heterostructures have drawn attention because of the existence of a quantum Hall state in graphene. SOC in this junction is not necessary, while strong SOC is required in semiconductor or TI/superconductor junctions. 253 Topologically protected Majorana bound state exists and may be detected by Andreev spectroscopy and Fraunhofer pattern anomalies. Although many endeavors have been made, ${ }^{253}$ further studies to realize topological superconductivity are expected.

\section{SUMMARY AND OUTLOOK}

To summarize, research to discover and verify TSCs has become one of the most active fields in condensed matter physics due to the potential applications in fault-tolerant topological quantum computation.1 Decades ago, the TSC candidates $\mathrm{Sr}_{2} \mathrm{RuO}_{4}{ }^{14|28| 36}$ and

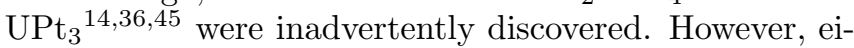
ther the chiral edge states or the MZMs in $\mathrm{Sr}_{2} \mathrm{RuO}_{4}$ have not been detected, and little evidence to support the spin-triplet pairing state for $\mathrm{UPt}_{3}$ has been found. Subsequently, research on topological materials became a hot topic, which opened up new horizons for exploring TSCs.1191 High pressure application is a relatively powerful method to induce superconductivity in TIs and semimetals, but the disadvantage of this technique is the difficulty of conducting further experiments to detect the pairing symmetry and gapless surface state. Therefore, the hard-tip contact method seems to provide a new approach to inducing superconductivity and simultaneously detecting the ZBP of gapless surface states.

The doping method offers an effective platform to study superconductivity in topological materials, and most experiments reveal the unique features associated with TSCs, such as spontaneous RSB, ZBPs, $170 \mid 171$ and MZMs 169 One of the application goals in studying TSCs is to realize topological quantum computation, and thus, many efforts have been made to fabricate devices. In this direction, the most progress has been made in probing MZMs in artificial heterostructures!7|14/213 In addition, more approaches to finding TSCs, such as natural heterostructures, $\frac{161}{2}$ gating-induced TSCs, $210 \mid 211$ or iron-based superconductors with nontrivial band structures, 254 are encouraged. The material systems with coexistence of topological bands and superconductivity, such as the layered compound $\mathrm{PbTaSe}_{2}$, 255|256, have become promising candidate systems for TSCs. Recently, thousands of topological (crystalline) insulators and topological semimetals have been theoretically classified.257.259 We believe that rapid development in the field of topological materials will greatly promote the investigation of topological superconductivity and motivate novel devices. 


\section{ACKNOWLEDGMENTS}

We thank Yi Zhou, Xin Lu, Yi Zheng, Guanghan Cao, and Fuchun Zhang for helpful discussions. This work was supported by the National Key R\&D Program of China (Grant No. 2016YFA0300402) and the National Science Foundation of China (Grant No. 11774305).

[1] C. Nayak, S. H. Simon, A. Stern, M. Freedman, S. Das Sarma, Rev. Mod. Phys. 2008, 80, 1083.

[2] G. E. Volovik, (Oxford: Oxford University Press) 2003.

[3] N. Read, D. Green, Phys. Rev. B 2000, 61, 10267.

[4] A. Y. Kitaev, Phys. Usp. 2001, 44, 131.

[5] E. Majorana, Il Nuovo Cimento 1937, 14, 171.

[6] F. Wilczek, Nat. Phys. 2009, 5, 614.

[7] C. W. J. Beenakker, Annu. Rev. Condens. Matter Phys. 2013, 4, 113.

[8] L. Fu, C. L. Kane, E. J. Mele, Phys. Rev. Lett. 2007, 98, 106803.

[9] L. Fu, C. L. Kane, Phys. Rev. B 2007, 76, 045302.

[10] L. Fu, C. L. Kane, Phys. Rev. Lett. 2008, 100, 096407.

[11] X.-L. Qi, S.-C. Zhang, Rev. Mod. Phys. 2011, 83, 1057.

[12] L. Fu, E. Berg, Phys. Rev. Lett. 2010, 105, 097001.

[13] J. D. Sau, R. M. Lutchyn, S. Tewari, S. Das Sarma, Phys. Rev. Lett. 2010, 104, 040502.

[14] M. Sato, Y. Ando, Rep. Prog. Phys. 2017, 80, 076501.

[15] Y. Ando, L. Fu, Annu. Rev. Condens. Matter Phys. 2015, 6, 361 .

[16] K. Ishida, H. Mukuda, Y. Kitaoka, K. Asayama, Z. Q. Mao, Y. Mori, Y. Maeno, Nature 1998, 396, 658.

[17] G. M. Luke, Y. Fudamoto, K. M. Kojima, M. I. Larkin, J. Merrin, B. Nachumi, Y. J. Uemura, Y. Maeno, Z. Q. Mao, Y. Mori, H. Nakamura, M. Sigrist, Nature 1998, 394,558 .

[18] G. M. Luke, A. Keren, L. P. Le, W. D. Wu, Y. J. Uemura, D. A. Bonn, L. Taillefer, J. D. Garrett, Phys. Rev. Lett. 1993, 71, 1466.

[19] V. Mourik, K. Zuo, S. M. Frolov, S. R. Plissard, E. P. A. M. Bakkers, L. P. Kouwenhoven, Science 2012, 336, 1003.

[20] J.-P. Xu, M.-X. Wang, Z. L. Liu, J.-F. Ge, X. Yang, C. Liu, Z. A. Xu, D. Guan, C. L. Gao, D. Qian, Y. Liu, Q.-H. Wang, F.-C. Zhang, Q.-K. Xue, J.-F. Jia, Phys. Rev. Lett. 2015, 114, 017001.

[21] H.-H. Sun, K.-W. Zhang, L.-H. Hu, C. Li, G.-Y. Wang, H.-Y. Ma, Z.-A. Xu, C.-L. Gao, D.-D. Guan, Y.-Y. Li, C. Liu, D. Qian, Y. Zhou, L. Fu, S.-C. Li, F.-C. Zhang, J.-F. Jia, Phys. Rev. Lett. 2016, 116, 257003.

[22] J. Wiedenmann, E. Bocquillon, R. S. Deacon, S. Hartinger, O. Herrmann, T. M. Klapwijk, L. Maier, C. Ames, C. Brüne, C. Gould, A. Oiwa, K. Ishibashi, S. Tarucha, H. Buhmann, L. W. Molenkamp, Nat. Commun. 2016, 7, 10303.

[23] R. S. Deacon, J. Wiedenmann, E. Bocquillon, F. Domínguez, T. M. Klapwijk, P. Leubner, C. Brüne, E. M. Hankiewicz, S. Tarucha, K. Ishibashi, H. Buhmann, L. W. Molenkamp, Phys. Rev. X 2017, 7, 021011.

[24] S. Hart, H. Ren, T. Wagner, P. Leubner, M. Mühlbauer, C. Brüne, H. Buhmann, L. W. Molenkamp, A. Yacoby,
Nat. Phys. 2014, 10, 638.

[25] J. F. Annett, (Oxford Master Series in Condensed Matter Physics, Oxford, 2004).

[26] C. C. Tsuei, J. R. Kirtley, Rev. Mod. Phys. 2000, 72, 969.

[27] G. R. Stewart, Rev. Mod. Phys. 2011, 83, 1589.

[28] Y. Maeno, S. Kittaka, T. Nomura, S. Yonezawa, K. Ishida, J. Phys. Soc. Jpn. 2012, 81, 011009.

[29] G.-H. Cao, J.-K. Bao, Z.-T. Tang, Y. Liu, H. Jiang, Philos. Mag. 2017, 97, 591.

[30] J. G. Bednorz, K. A. Müller, Z. Phys. B 1986, 64, 189.

[31] Y. Maeno, H. Hashimoto, K. Yoshida, S. Nishizaki, T. Fujita, J. G. Bednorz, F. Lichtenberg, Nature 1994, 372, 532.

[32] A. P. Mackenzie, Y. Maeno, Rev. Mod. Phys. 2003, 75, 657.

[33] A. M. Clogston, Phys. Rev. Lett. 1962, 9, 266.

[34] K. D. Nelson, Z. Q. Mao, Y. Maeno, Y. Liu, Science 2004, 306, 1151

[35] V. B. Geshkenbein, A. I. Larkin, A. Barone, Phys. Rev. $B$ 1987, 36, 235 .

[36] C. Kallin, J. Berlinsky, Rep. Prog. Phys. 2016, 79, 054502 .

[37] F. Kidwingira, J. D. Strand, D. J. Van Harlingen, Y. Maeno, Science 2006, 314, 1267.

[38] J. Jang, D. G. Ferguson, V. Vakaryuk, R. Budakian, S. B. Chung, P. M. Goldbart, Y. Maeno, Science 2011, 331, 186.

[39] N. B. Kopnin, M. M. Salomaa, Phys. Rev. B 1991, 44, 9667.

[40] S. Kashiwaya, H. Kashiwaya, H. Kambara, T. Furuta, H. Yaguchi, Y. Tanaka, Y. Maeno, Phys. Rev. Lett. 2011, 10\%, 077003.

[41] C. Kallin, A. J. Berlinsky, J. Phys.-Condes. Matter 2009, 21, 164210.

[42] A. Pustogow, Y. Luo, A. Chronister, Y. S. Su, D. A. Sokolov, F. Jerzembeck, A. P. Mackenzie, C. W. Hicks, N. Kikugawa, S. Raghu, E. D. Bauer, S. E. Brown, arXiv:1904.00047.

[43] A. P. Schnyder, P. M. R. Brydon, J. Phys.-Condes. Matter 2015, 27, 243201.

[44] Y. Ueno, A. Yamakage, Y. Tanaka, M. Sato, Phys. Rev. Lett. 2013, 111, 087002.

[45] R. Joynt, L. Taillefer, Rev. Mod. Phys. 2002, 74, 235.

[46] E. R. Schemm, W. J. Gannon, C. M. Wishne, W. P. Halperin, A. Kapitulnik, Science 2014, 345, 190.

[47] Y. Tsutsumi, M. Ishikawa, T. Kawakami, T. Mizushima, M. Sato, M. Ichioka, K. Machida, J. Phys. Soc. Jap. 2013, 82, 113707.

[48] Y. Machida, A. Itoh, Y. So, K. Izawa, Y. Haga, E. Yamamoto, N. Kimura, Y. Onuki, Y. Tsutsumi, K. Machida, Phys. Rev. Lett. 2012, 108, 157002. 
[49] J. D. Strand, D. J. Bahr, D. J. Van Harlingen, J. P. Davis, W. J. Gannon, W. P. Halperin, Science 2010, 328, 1368.

[50] Y. Tsutsumi, M. Ishikawa, T. Kawakami, T. Mizushima, M. Sato, M. Ichioka, K. Machida, J. Phys. Soc. Jpn 2013, 82, 113707.

[51] S. Kobayashi, Y. Yanase, M. Sato, Phys. Rev. B 2016, 94, 134512.

[52] G. R. Stewart, Rev. Mod. Phys. 2011, 83, 1589.

[53] N. Hao, J. Hu, Natl. Sci. Rev. 2019, 6, 213.

[54] N. Hao, J. Hu, Phys. Rev. X 2014, 4, 031053.

[55] Q.-Y. Wang, Z. Li, W.-H. Zhang, Z.-C. Zhang, J.-S. Zhang, W. Li, H. Ding, Y.-B. Ou, P. Deng, K. Chang, J. Wen, C.-L. Song, K. He, J.-F. Jia, S.-H. Ji, Y.-Y. Wang, L.-L. Wang, X. Chen, X.-C. Ma, Q.-K. Xue, Chin. Phys. Lett. 2012, 29, 037402.

[56] Z. F. Wang, H. Zhang, D. Liu, C. Liu, C. Tang, C. Song, Y. Zhong, J. Peng, F. Li, C. Nie, L. Wang, X. J. Zhou, X. Ma, Q. K. Xue, F. Liu, Nat. Mater. 2016, 15, 968.

[57] X. Shi, Z. Han, P. Richard, X. Wu, X. Peng, T. Qian, S. Wang, J. Hu, Y. Sun, H. Ding, Sci. Bull. 2017, 62, 503.

[58] X. Wu, S. Qin, Y. Liang, H. Fan, J. Hu, Phys. Rev. B 2016, 93, 115129.

[59] D. Wang, L. Kong, P. Fan, H. Chen, S. Zhu, W. Liu, L. Cao, Y. Sun, S. Du, J. Schneeloch, R. Zhong, G. Gu, L. Fu, H. Ding, H.-J. Gao, Science 2018, 362, 333.

[60] M. Chen, X. Chen, H. Yang, Z. Du, X. Zhu, E. Wang, H.-H. Wen, Nat. Commun. 2018, 9, 970.

[61] X. F. Lu, N. Z. Wang, H. Wu, Y. P. Wu, D. Zhao, X. Z. Zeng, X. G. Luo, T. Wu, W. Bao, G. H. Zhang, F. Q. Huang, Q. Z. Huang, X. H. Chen, Nat. Mater. 2015, 14,325 .

[62] F.-C. Hsu, J.-Y. Luo, K.-W. Yeh, T.-K. Chen, T.-W. Huang, P. M. Wu, Y.-C. Lee, Y.-L. Huang, Y.-Y. Chu, D.-C. Yan, M.-K. Wu, Proc. Natl. Acad. Sci. U.S.A. 2008, 105, 14262.

[63] M. Smidman, G. M. Pang, H. X. Zhou, N. Z. Wang, W. Xie, Z. F. Weng, Y. Chen, X. L. Dong, X. H. Chen, Z. X. Zhao, H. Q. Yuan, Phys. Rev. B 2017, 96, 014504.

[64] X. H. Niu, R. Peng, H. C. Xu, Y. J. Yan, J. Jiang, D. F. Xu, T. L. Yu, Q. Song, Z. C. Huang, Y. X. Wang, B. P. Xie, X. F. Lu, N. Z. Wang, X. H. Chen, Z. Sun, D. L. Feng, Phys. Rev. B 2015, 92, 060504.

[65] Q. Liu, C. Chen, T. Zhang, R. Peng, Y.-J. Yan, C.-H.P. Wen, X. Lou, Y.-L. Huang, J.-P. Tian, X.-L. Dong, G.-W. Wang, W.-C. Bao, Q.-H. Wang, Z.-P. Yin, Z.-X. Zhao, D.-L. Feng, Phys. Rev. X 2018, 8, 041056.

[66] C. Caroli, P. G. De Gennes, J. Matricon, Phys. Lett. 1964, 9, 307.

[67] G. E. Volovik, Turk. J. Phys. 1996, 20, 697.

[68] Z. Du, X. Yang, D. Altenfeld, Q. Gu, H. Yang, I. Eremin, P. J. Hirschfeld, I. I. Mazin, H. Lin, X. Zhu, H.-H. Wen, Nat. Phys. 2018, 14, 134.

[69] P. J. Hirschfeld, M. M. Korshunov, I. I. Mazin, Rep. Prog. Phys. 2011, 74, 124508.

[70] P. Zhang, X. Wu, K. Yaji, G. Dai, X. Wang, C. Jin, J. Hu, R. Thomale, T. Kondo, S. Shin, arXiv:1803.00846.

[71] X. Wu, S. Qin, Y. Liang, C. Le, H. Fan, J. Hu, Phys. Rev. B 2015, 91, 081111.

[72] J.-K. Bao, J.-Y. Liu, C.-W. Ma, Z.-H. Meng, Z.-T. Tang, Y.-L. Sun, H.-F. Zhai, H. Jiang, H. Bai, C.-M. Feng, Z.-A. Xu, G.-H. Cao, Phys. Rev. X 2015, 5,
011013

[73] Z.-T. Tang, J.-K. Bao, Y. Liu, Y.-L. Sun, A. Ablimit, H.-F. Zhai, H. Jiang, C.-M. Feng, Z.-A. Xu, G.-H. Cao, Phys. Rev. B 2015, 91, 020506.

[74] Z.-T. Tang, J.-K. Bao, Z. Wang, H. Bai, H. Jiang, Y. Liu, H.-F. Zhai, C.-M. Feng, Z.-A. Xu, G.-H. Cao, Sci. China-Mater. 2015, 58, 16.

[75] Q.-G. Mu, B.-B. Ruan, B.-J. Pan, T. Liu, J. Yu, K. Zhao, G.-F. Chen, Z.-A. Ren, Phys. Rev. Materials 2018, 2, 034803.

[76] F. F. Balakirev, T. Kong, M. Jaime, R. D. McDonald, C. H. Mielke, A. Gurevich, P. C. Canfield, S. L. Bud'ko, Phys. Rev. B 2015, 91, 220505.

[77] H. Zuo, J.-K. Bao, Y. Liu, J. Wang, Z. Jin, Z. Xia, L. Li, Z. Xu, J. Kang, Z. Zhu, G.-H. Cao, Phys. Rev. B 2017, 95, 014502.

[78] J. Yang, Z. T. Tang, G. H. Cao, G.-Q. Zheng, Phys. Rev. Lett. 2015, 115, 147002.

[79] H. Z. Zhi, T. Imai, F. L. Ning, J.-K. Bao, G.-H. Cao, Phys. Rev. Lett. 2015, 114, 147004.

[80] D. T. Adroja, A. Bhattacharyya, M. Telling, Y. Feng, M. Smidman, B. Pan, J. Zhao, A. D. Hillier, F. L. Pratt, A. M. Strydom, Phys. Rev. B 2015, 92, 134505.

[81] G. M. Pang, M. Smidman, W. B. Jiang, J. K. Bao, Z. F. Weng, Y. F. Wang, L. Jiao, J. L. Zhang, G. H. Cao, H. Q. Yuan, Phys. Rev. B 2015, 91, 220502.

[82] A. Jayaraman, Rev. Mod. Phys. 1983, 55, 65.

[83] H.-K. Mao, X.-J. Chen, Y. Ding, B. Li, L. Wang, Rev. Mod. Phys. 2018, 90, 015007.

[84] A. P. Drozdov, M. I. Eremets, I. A. Troyan, V. Ksenofontov, S. I. Shylin, Nature 2015, 525, 73.

[85] L. P. Gor'kov, V. Z. Kresin, Rev. Mod. Phys. 2018, 90, 011001.

[86] A. Roth, C. Brüne, H. Buhmann, L. W. Molenkamp, J. Maciejko, X.-L. Qi, S.-C. Zhang, Science 2009, 325, 294.

[87] D. Hsieh, D. Qian, L. Wray, Y. Xia, Y. S. Hor, R. J. Cava, M. Z. Hasan, Nature 2008, 452, 970.

[88] Y. L. Chen, J. G. Analytis, J.-H. Chu, Z. K. Liu, S.-K. Mo, X. L. Qi, H. J. Zhang, D. H. Lu, X. Dai, Z. Fang, S. C. Zhang, I. R. Fisher, Z. Hussain, Z.-X. Shen, Science 2009, 325, 178.

[89] Y. Xia, D. Qian, D. Hsieh, L. Wray, A. Pal, H. Lin, A. Bansil, D. Grauer, Y. S. Hor, R. J. Cava, M. Z. Hasan, Nat. Phys. 2009, 5, 398.

[90] D. Hsieh, Y. Xia, D. Qian, L. Wray, F. Meier, J. H. Dil, J. Osterwalder, L. Patthey, A. V. Fedorov, H. Lin, A. Bansil, D. Grauer, Y. S. Hor, R. J. Cava, M. Z. Hasan, Phys. Rev. Lett. 2009, 103, 146401.

[91] M. Z. Hasan, C. L. Kane, Rev. Mod. Phys. 2010, 82, 3045.

[92] J. L. Zhang, S. J. Zhang, H. M. Weng, W. Zhang, L. X. Yang, Q. Q. Liu, S. M. Feng, X. C. Wang, R. C. Yu, L. Z. Cao, L. Wang, W. G. Yang, H. Z. Liu, W. Y. Zhao, S. C. Zhang, X. Dai, Z. Fang, C. Q. Jin, Proc. Natl. Acad. Sci. U.S.A. 2011, 108, 24.

[93] C. Zhang, L. Sun, Z. Chen, X. Zhou, Q. Wu, W. Yi, J. Guo, X. Dong, Z. Zhao, Phys. Rev. B 2011, 83, 140504 .

[94] K. Matsubayashi, T. Terai, J. S. Zhou, Y. Uwatoko, Phys. Rev. B 2014, 90, 125126.

[95] K. Kirshenbaum, P. S. Syers, A. P. Hope, N. P. Butch, J. R. Jeffries, S. T. Weir, J. J. Hamlin, M. B. Maple, Y. K. Vohra, J. Paglione, Phys. Rev. Lett. 2013, 111, 
087001.

[96] J. Zhu, J. L. Zhang, P. P. Kong, S. J. Zhang, X. H. Yu, J. L. Zhu, Q. Q. Liu, X. Li, R. C. Yu, R. Ahuja, W. G. Yang, G. Y. Shen, H. K. Mao, H. M. Weng, X. Dai, Z. Fang, Y. S. Zhao, C. Q. Jin, Sci. Rep. 2013, 3, 2016.

[97] J. Zhao, H. Liu, L. Ehm, Z. Chen, S. Sinogeikin, Y. Zhao, G. Gu, Inorg. Chem. 2011, 50, 11291.

[98] Y. Qi, W. Shi, P. G. Naumov, N. Kumar, R. Sankar, W. Schnelle, C. Shekhar, F.-C. Chou, C. Felser, B. Yan, S. A. Medvedev, Adv. Mater. 2017, 29, 1605965.

[99] J.-J. Ying, V. V. Struzhkin, Z.-Y. Cao, A. F. Goncharov, H.-K. Mao, F. Chen, X.-H. Chen, A. G. Gavriliuk, X.-J. Chen, Phys. Rev. B 2016, 93, 100504.

[100] L. P. He, Y. T. Jia, S. J. Zhang, X. C. Hong, C. Q. Jin, S. Y. Li, npj Quantum Materials 2016, 1, 16014.

[101] Y. Zhou, J. Wu, W. Ning, N. Li, Y. Du, X. Chen, R. Zhang, Z. Chi, X. Wang, X. Zhu, P. Lu, C. Ji, X. Wan, Z. Yang, J. Sun, W. Yang, M. Tian, Y. Zhang, H.-k. Mao, Proc. Natl. Acad. Sci. U.S.A. 2016, 113, 2904.

[102] Y. Qi, W. Shi, P. G. Naumov, N. Kumar, W. Schnelle, O. Barkalov, C. Shekhar, H. Borrmann, C. Felser, B. Yan, S. A. Medvedev, Phys. Rev. B 2016, 94, 054517.

[103] Y. Li, Y. Zhou, Z. Guo, F. Han, X. Chen, P. Lu, X. Wang, C. An, Y. Zhou, J. Xing, G. Du, X. Zhu, H. Yang, J. Sun, Z. Yang, W. Yang, H.-K. Mao, Y. Zhang, H.-H. Wen, npj Quantum Materials 2017, 2, 66 .

[104] Y. Qi, P. G. Naumov, M. N. Ali, C. R. Rajamathi, W. Schnelle, O. Barkalov, M. Hanfland, S.-C. Wu, C. Shekhar, Y. Sun, V. Süß, M. Schmidt, U. Schwarz, E. Pippel, P. Werner, R. Hillebrand, T. Förster, E. Kampert, S. Parkin, R. J. Cava, C. Felser, B. Yan, S. A. Medvedev, Nat. Commun. 2016, \%, 11038.

[105] F. F. Tafti, M. S. Torikachvili, R. L. Stillwell, B. Baer, E. Stavrou, S. T. Weir, Y. K. Vohra, H.-Y. Yang, E. F. McDonnell, S. K. Kushwaha, Q. D. Gibson, R. J. Cava, J. R. Jeffries, Phys. Rev. B 2017, 95, 014507.

[106] D. Kang, Y. Zhou, W. Yi, C. Yang, J. Guo, Y. Shi, S. Zhang, Z. Wang, C. Zhang, S. Jiang, A. Li, K. Yang, Q. Wu, G. Zhang, L. L. Sun, Z. X. Zhao, Nat. Commun. 2015, 6, 7804 .

[107] X. C. Pan, X. L. Chen, H. M. Liu, Y. Q. Feng, Z. X. Wei, Y. H. Zhou, Z. H. Chi, L. Pi, F. Yen, F. Q. Song, X. G. Wan, Z. R. Yang, B. G. Wang, G. H. Wang, Y. H. Zhang, Nat. Commun. 2015, 6, 7805.

[108] Z. Chi, X. Chen, C. An, L. Yang, J. Zhao, Z. Feng, Y. Zhou, Y. Zhou, C. Gu, B. Zhang, Y. Yuan, C. Kenney-Benson, W. Yang, G. Wu, X. Wan, Y. Shi, X. Yang, Z. Yang, npj Quantum Materials 2018, 3, 28.

[109] Y. Li, C. An, C. Hua, X. Chen, Y. Zhou, Y. Zhou, R. Zhang, C. Park, Z. Wang, Y. Lu, Y. Zheng, Z. Yang, Z.-A. Xu, npj Quantum Materials 2018, 3, 58.

[110] Y. S. Hor, A. J. Williams, J. G. Checkelsky, P. Roushan, J. Seo, Q. Xu, H. W. Zandbergen, A. Yazdani, N. P. Ong, R. J. Cava, Phys. Rev. Lett. 2010, 104, 057001.

[111] Shruti, V. K. Maurya, P. Neha, P. Srivastava, S. Patnaik, Phys. Rev. B 2015, 92, 020506.

[112] R. Vilaplana, D. Santamaría-Pérez, O. Gomis, F. J. Manjón, J. González, A. Segura, A. Muñoz, P. Rodríguez-Hernández, E. Pérez-González, V. MarínBorrás, V. Muñoz-Sanjose, C. Drasar, V. Kucek, Phys. Rev. B 2011, 84, 184110.
[113] K. Ishizaka, M. S. Bahramy, H. Murakawa, M. Sakano, T. Shimojima, T. Sonobe, K. Koizumi, S. Shin, H. Miyahara, A. Kimura, K. Miyamoto, T. Okuda, H. Namatame, M. Taniguchi, R. Arita, N. Nagaosa, K. Kobayashi, Y. Murakami, R. Kumai, Y. Kaneko, Y. Onose, Y. Tokura, Nat. Mater. 2011, 10, 521.

[114] M. Sakano, M. S. Bahramy, A. Katayama, T. Shimojima, H. Murakawa, Y. Kaneko, W. Malaeb, S. Shin, K. Ono, H. Kumigashira, R. Arita, N. Nagaosa, H. Y. Hwang, Y. Tokura, K. Ishizaka, Phys. Rev. Lett. 2013, 110, 107204.

[115] Y. L. Chen, M. Kanou, Z. K. Liu, H. J. Zhang, J. A. Sobota, D. Leuenberger, S. K. Mo, B. Zhou, S.-L. Yang, P. S. Kirchmann, D. H. Lu, R. G. Moore, Z. Hussain, Z. X. Shen, X. L. Qi, T. Sasagawa, Nat. Phys. 2013, 9, 704 .

[116] Z. K. Liu, B. B. Zhou, Y. Zhang, Z. J. Wang, H. Z. Weng, D. Prabhakaran, S. K. Mo, Z. X. Shen, Z. Fang, X. Dai, Z. Hussain, Y. L. Chen, Science 2014, 343, 864.

[117] Z. K. Liu, J. Jiang, B. Zhou, Z. J. Wang, Y. Zhang, H. M. Weng, D. Prabhakaran, S.-K. Mo, H. Peng, P. Dudin, T. Kim, M. Hoesch, Z. Fang, X. Dai, Z. X. Shen, D. L. Feng, Z. Hussain, Y. L. Chen, Nat. Mater. 2014, 13, 677 .

[118] Q. Li, D. E. Kharzeev, C. Zhang, Y. Huang, I. Pletikosić, A. V. Fedorov, R. D. Zhong, J. A. Schneeloch, G. D. Gu, T. Valla, Nat. Phys. 2016, 12, 550.

[119] A. V. X. G. Wan, A. M. Turner, S. Y. Savrasov, Phys. Rev. B 2011, 83, 205101.

[120] H. Weng, C. Fang, Z. Fang, B. A. Bernevig, X. Dai, Phys. Rev. X 2015, 5, 011029.

[121] S.-Y. Xu, I. Belopolski, N. Alidoust, M. Neupane, G. Bian, C. Zhang, R. Sankar, G. Chang, Z. Yuan, C.C. Lee, S.-M. Huang, H. Zheng, J. Ma, D. S. Sanchez, B. Wang, A. Bansil, F. Chou, P. P. Shibayev, H. Lin, S. Jia, M. Z. Hasan, Science 2015, 349, 613.

[122] S.-Y. Xu, I. Belopolski, D. S. Sanchez, C. Zhang, G. Chang, C. Guo, G. Bian, Z. Yuan, H. Lu, T.-R. Chang, P. P. Shibayev, M. L. Prokopovych, N. Alidoust, H. Zheng, C.-C. Lee, S.-M. Huang, R. Sankar, F. Chou, C.-H. Hsu, H.-T. Jeng, A. Bansil, T. Neupert, V. N. Strocov, H. Lin, S. Jia, M. Z. Hasan, Sci. Adv. 2015, 1, e1501092.

[123] S.-Y. Xu, N. Alidoust, I. Belopolski, Z. Yuan, G. Bian, T.-R. Chang, H. Zheng, V. N. Strocov, D. S. Sanchez, G. Chang, C. Zhang, D. Mou, Y. Wu, L. Huang, C.C. Lee, S.-M. Huang, B. Wang, A. Bansil, H.-T. Jeng, T. Neupert, A. Kaminski, H. Lin, S. Jia, M. Zahid Hasan, Nat. Phys. 2015, 11, 748.

[124] D.-F. Xu, Y.-P. Du, Z. Wang, Y.-P. Li, X.-H. Niu, Q. Yao, P. Dudin, Z.-A. Xu, X.-G. Wan, D.-L. Feng, Chin. Phys. Lett. 2015, 32, 107101.

[125] Z. Wang, Y. Zheng, Z. Shen, Y. Lu, H. Fang, F. Sheng, Y. Zhou, X. Yang, Y. Li, C. Feng, Z.-A. Xu, Phys. Rev. B 2016, 93, 121112.

[126] J. Hu, T. F. Rosenbaum, J. B. Betts, Phys. Rev. Lett. 2005, 95, 186603.

[127] R. D. dos Reis, M. O. Ajeesh, N. Kumar, F. Arnold, C. Shekhar, M. Naumann, M. Schmidt, M. Nicklas, E. Hassinger, New J. Phys. 2016, 18, 085006.

[128] Y. Li, Z. Wang, P. Li, X. Yang, Z. Shen, F. Sheng, X. Li, Y. Lu, Y. Zheng, Z.-A. Xu, Front. Phys. 2017, 12, 127205 . 
[129] S. Zhang, Q. Wu, L. Schoop, M. N. Ali, Y. Shi, N. Ni, Q. Gibson, S. Jiang, V. Sidorov, W. Yi, J. Guo, Y. Zhou, D. Wu, P. Gao, D. Gu, C. Zhang, S. Jiang, K. Yang, A. Li, Y. Li, X. Li, J. Liu, X. Dai, Z. Fang, R. J. Cava, L. Sun, Z. Zhao, Phys. Rev. B 2015, 91, 165133.

[130] S. Kobayashi, M. Sato, Phys. Rev. Lett. 2015, 115, 187001.

[131] J. D. Sau, S. Tewari, Phys. Rev. B 2012, 86, 104509.

[132] A. Chen, M. Franz, Phys. Rev. B 2016, 93, 201105.

[133] J. Zhang, F.-L. Liu, J.-K. Dong, Y. Xu, N.-N. Li, W.-G. Yang, S.-Y. Li, Chin. Phys. Lett. 2015, 32, 097102.

[134] Y. Wu, D. Mou, N. H. Jo, K. Sun, L. Huang, S. L. Bud'ko, P. C. Canfield, A. Kaminski, Phys. Rev. B 2016, 94, 121113.

[135] K. Deng, G. Wan, P. Deng, K. Zhang, S. Ding, E. Wang, M. Yan, H. Huang, H. Zhang, Z. Xu, J. Denlinger, A. Fedorov, H. Yang, W. Duan, H. Yao, Y. Wu, S. Fan, H. Zhang, X. Chen, S. Zhou, Nat. Phys. 2016, 12, 1105.

[136] T.-R. Chang, S.-Y. Xu, G. Chang, C.-C. Lee, S.-M. Huang, B. Wang, G. Bian, H. Zheng, D. S. Sanchez, I. Belopolski, N. Alidoust, M. Neupane, A. Bansil, H.T. Jeng, H. Lin, M. Zahid Hasan, Nat. Commun. 2016, 7, 10639 .

[137] G. Chang, S.-Y. Xu, D. S. Sanchez, S.-M. Huang, C.C. Lee, T.-R. Chang, G. Bian, H. Zheng, I. Belopolski, N. Alidoust, H.-T. Jeng, A. Bansil, H. Lin, M. Z. Hasan, Sci. Adv. 2016, 2, e1600295.

[138] A. A. Soluyanov, D. Gresch, Z. Wang, Q. Wu, M. Troyer, X. Dai, B. A. Bernevig, Nature 2015, 527, 495.

[139] M. N. Ali, J. Xiong, S. Flynn, J. Tao, Q. D. Gibson, L. M. Schoop, T. Liang, N. Haldolaarachchige, M. Hirschberger, N. P. Ong, R. J. Cava, Nature 2014, 514, 205.

[140] K. Deng, G. Wan, P. Deng, K. Zhang, S. Ding, E. Wang, M. Yan, H. Huang, H. Zhang, Z. Xu, J. Denlinger, A. Fedorov, H. Yang, W. Duan, H. Yao, Y. Wu, S. Fan, H. Zhang, X. Chen, S. Zhou, Nat. Phys. 2016, 12, 1105.

[141] L. Huang, T. M. McCormick, M. Ochi, Z. Zhao, M.T. Suzuki, R. Arita, Y. Wu, D. Mou, H. Cao, J. Yan, N. Trivedi, A. Kaminski, Nat. Mater. 2016, 15, 1155.

[142] J. Nayak, S.-C. Wu, N. Kumar, C. Shekhar, S. Singh, J. Fink, E. E. D. Rienks, G. H. Fecher, S. S. P. Parkin, B. Yan, C. Felser, Nat. Commun. 2017, 8, 13942.

[143] B. Q. Lv, Z.-L. Feng, Q.-N. Xu, X. Gao, J.-Z. Ma, L.-Y. Kong, P. Richard, Y.-B. Huang, V. N. Strocov, C. Fang, H.-M. Weng, Y.-G. Shi, T. Qian, H. Ding, Nature 2017 546,627 .

[144] Y. Shao, Z. Sun, Y. Wang, C. Xu, R. Sankar, A. J. Breindel, C. Cao, M. M. Fogler, A. J. Millis, F. Chou, Z. Li, T. Timusk, M. B. Maple, D. N. Basov, Proc. Natl. Acad. Sci. U.S.A. 2019, 116, 1168.

[145] Y. P. Li, Z. Wang, Y. H. Lu, X. J. Yang, Z. X. Shen, F. Sheng, C. M. Feng, Y. Zheng, Z. A. Xu, arXiv:1603.04056

[146] B. Shen, X. Deng, G. Kotliar, N. Ni, Phys. Rev. B 2016, 93, 195119.

[147] D. Gresch, Q. Wu, G. W. Winkler, A. A. Soluyanov, New. J. Phys. 2017, 19, 035001.

[148] H. Wang, L. Ma, J. Wang, Sci. Bull. 2018, 63, 1141.

[149] H. Wang, H. Wang, H. Liu, H. Lu, W. Yang, S. Jia, X.J. Liu, X. C. Xie, J. Wei, J. Wang, Nat. Mater. 2016, 15,38 .
[150] L. Aggarwal, A. Gaurav, G. S. Thakur, Z. Haque, A. K. Ganguli, G. Sheet, Nat. Mater. 2016, 15, 32.

[151] Y. Kamihara, T. Watanabe, M. Hirano, H. Hosono, J. Am. Chem. Soc. 2008, 130, 3296.

[152] S. Jiang, H. Xing, G. Xuan, C. Wang, Z. Ren, C. Feng, J. Dai, Z. Xu, G. Cao, J. Phys.: Condens. Matter. 2009, 21, 382203.

[153] C.-Z. Chang, J. Zhang, X. Feng, J. Shen, Z. Zhang, M. Guo, K. Li, Y. Ou, P. Wei, L.-L. Wang, Z.-Q. Ji, Y. Feng, S. Ji, X. Chen, J. Jia, X. Dai, Z. Fang, S.-C. Zhang, K. He, Y. Wang, L. Lu, X.-C. Ma, Q.-K. Xue, Science 2013, 340, 167.

[154] T. Dietl, Nat. Mater. 2010, 9, 965.

[155] S. Sasaki, T. Mizushima, Physica C 2015, 514, 206.

[156] M. Kriener, K. Segawa, Z. Ren, S. Sasaki, S. Wada, S. Kuwabata, Y. Ando, Phys. Rev. B 2011, 84, 054513.

[157] Z. Liu, X. Yao, J. Shao, M. Zuo, L. Pi, S. Tan, C. Zhang, Y. Zhang, J. Am. Chem. Soc. 2015, 137, 10512.

[158] Y. Qiu, K. Nocona Sanders, J. Dai, J. E. Medvedeva, W. Wu, P. Ghaemi, T. Vojta, Y. San Hor, arXiv:1512.03519 .

[159] Z. Wang, A. A. Taskin, T. Frölich, M. Braden, Y. Ando, Chem. Mater. 2016, 28, 779.

[160] C. X. Trang, Z. Wang, D. Takane, K. Nakayama, S. Souma, T. Sato, T. Takahashi, A. A. Taskin, Y. Ando, Phys. Rev. B 2016, 93, 241103.

[161] S. Sasaki, K. Segawa, Y. Ando, Phys. Rev. B 2014, 90, 220504

[162] A. S. Erickson, J.-H. Chu, M. F. Toney, T. H. Geballe, I. R. Fisher, Phys. Rev. B 2009, 79, 024520.

[163] G. Balakrishnan, L. Bawden, S. Cavendish, M. R. Lees, Phys. Rev. B 2013, 87, 140507.

[164] R. D. Zhong, J. A. Schneeloch, T. S. Liu, F. E. Camino, J. M. Tranquada, G. D. Gu, Phys. Rev. B 2014, 90, 020505 .

[165] M. Kriener, K. Segawa, Z. Ren, S. Sasaki, Y. Ando, Phys. Rev. Lett. 2011, 106, 127004.

[166] N. Levy, T. Zhang, J. Ha, F. Sharifi, A. A. Talin, Y. Kuk, J. A. Stroscio, Phys. Rev. Lett. 2013, 110, 117001.

[167] E. Lahoud, E. Maniv, M. S. Petrushevsky, M. Naamneh, A. Ribak, S. Wiedmann, L. Petaccia, Z. Salman, K. B. Chashka, Y. Dagan, A. Kanigel, Phys. Rev. B 2013, 88, 195107.

[168] T. V. Bay, T. Naka, Y. K. Huang, H. Luigjes, M. S. Golden, A. de Visser, Phys. Rev. Lett. 2012, 108, 057001 .

[169] S. Sasaki, M. Kriener, K. Segawa, K. Yada, Y. Tanaka, M. Sato, Y. Ando, Phys. Rev. Lett. 2011, 107, 217001.

[170] K. Matano, M. Kriener, K. Segawa, Y. Ando, G.-Q. Zheng, Nat. Phys. 2016, 12, 852.

[171] S. Yonezawa, K. Tajiri, S. Nakata, Y. Nagai, Z. Wang, K. Segawa, Y. Ando, Y. Maeno, Nat. Phys. 2017, 13, 123.

[172] R. Tao, Y.-J. Yan, X. Liu, Z.-W. Wang, Y. Ando, Q.-H. Wang, T. Zhang, D.-L. Feng, Phys. Rev. X 2018, 8, 041024

[173] L. Fu, Phys. Rev. B 2014, 90, 100509.

[174] K. Nakayama, K. Eto, Y. Tanaka, T. Sato, S. Souma, T. Takahashi, K. Segawa, Y. Ando, Phys. Rev. Lett. 2012, 109, 236804.

[175] K. Nakayama, H. Kimizuka, Y. Tanaka, T. Sato, S. Souma, T. Takahashi, S. Sasaki, K. Segawa, Y. Ando, Phys. Rev. B 2015, 92, 100508. 
[176] Y. Pan, A. M. Nikitin, G. K. Araizi, Y. K. Huang, Y. Matsushita, T. Naka, A. de Visser, Sci. Rep. 2016, 6, 28632.

[177] G. Du, Y. Li, J. Schneeloch, R. D. Zhong, G. Gu, H. Yang, H. Lin, H.-H. Wen, Sci. China Phys. Mech. Astron. 2017, 60, 37411.

[178] T. Asaba, B. J. Lawson, C. Tinsman, L. Chen, P. Corbae, G. Li, Y. Qiu, Y. S. Hor, L. Fu, L. Li, Phys. Rev. $X$ 2017, 7, 011009.

[179] J. Shen, W.-Y. He, N. F. Q. Yuan, Z. Huang, C.-w. Cho, S. H. Lee, Y. S. Hor, K. T. Law, R. Lortz, npj Quantum Materials 2017, 2, 59.

[180] L. Andersen, Z. Wang, T. Lorenz, Y. Ando, Phys. Rev. $B$ 2018, 98, 220512.

[181] L. Fu, E. Berg, Phys. Rev. Lett. 2010, 105, 097001.

[182] X. G. Wan, S. Y. Savrasov, Nat. Commun. 2014, 5, 4144.

[183] L. Fu, Phys. Rev. Lett. 2011, 106, 106802.

[184] T. H. Hsieh, H. Lin, J. Liu, W. Duan, A. Bansil, L. Fu, Nat. Commun. 2012, 3, 982.

[185] Y. Tanaka, Z. Ren, T. Sato, K. Nakayama, S. Souma, T. Takahashi, K. Segawa, Y. Ando, Nat. Phys. 2012, 8, 800.

[186] L. P. He, Z. Zhang, J. Pan, X. C. Hong, S. Y. Zhou, S. Y. Li, Phys. Rev. B 2013, 88, 014523.

[187] M. Novak, S. Sasaki, M. Kriener, K. Segawa, Y. Ando, Phys. Rev. B 2013, 88, 140502.

[188] M. Saghir, J. A. T. Barker, G. Balakrishnan, A. D. Hillier, M. R. Lees, Phys. Rev. B 2014, 90, 064508.

[189] S. Sasaki, Z. Ren, A. A. Taskin, K. Segawa, L. Fu, Y. Ando, Phys. Rev. Lett. 2012, 109, 217004.

[190] T. Sato, Y. Tanaka, K. Nakayama, S. Souma, T. Takahashi, S. Sasaki, Z. Ren, A. A. Taskin, K. Segawa, Y. Ando, Phys. Rev. Lett. 2013, 110, 206804.

[191] Y. Tanaka, T. Sato, K. Nakayama, S. Souma, T. Takahashi, Z. Ren, M. Novak, K. Segawa, Y. Ando, Phys. Rev. B 2013, 87, 155105.

[192] Y. Tanaka, Z. Ren, T. Sato, K. Nakayama, S. Souma, T. Takahashi, K. Segawa, Y. Ando, Nat. Phys. 2012, 8, 800 .

[193] P. Dziawa, B. J. Kowalski, K. Dybko, R. Buczko, A. Szczerbakow, M. Szot, E. Łusakowska, T. Balasubramanian, B. M. Wojek, M. H. Berntsen, O. Tjernberg, T. Story, Nat. Mater. 2012, 11, 1023.

[194] T. Liang, Q. Gibson, J. Xiong, M. Hirschberger, S. P. Koduvayur, R. J. Cava, N. P. Ong, Nat. Commun. 2013, 4, 2696.

[195] G. Du, Z. Du, D. Fang, H. Yang, R. D. Zhong, J. Schneeloch, G. D. Gu, H.-H. Wen, Phys. Rev. B 2015, 92, 020512.

[196] C. H. Ahn, A. Bhattacharya, M. Di Ventra, J. N. Eckstein, C. D. Frisbie, M. E. Gershenson, A. M. Goldman, I. H. Inoue, J. Mannhart, A. J. Millis, A. F. Morpurgo, D. Natelson, J.-M. Triscone, Rev. Mod. Phys. 2006, 78, 1185.

[197] Y. Saito, T. Nojima, Y. Iwasa, Nat. Rev. Mater. 2017, 2, 16094.

[198] Y. Saito, T. Nojima, Y. Iwasa, Supercond. Sci. Technol. 2016, 29, 093001.

[199] B. Lei, N. Z. Wang, C. Shang, F. B. Meng, L. K. Ma, X. G. Luo, T. Wu, Z. Sun, Y. Wang, Z. Jiang, B. H. Mao, Z. Liu, Y. J. Yu, Y. B. Zhang, X. H. Chen, Phys. Rev. B 2017, 95, 020503.
[200] C. S. Zhu, J. H. Cui, B. Lei, N. Z. Wang, C. Shang, F. B. Meng, L. K. Ma, X. G. Luo, T. Wu, Z. Sun, X. H. Chen, Phys. Rev. B 2017, 95, 174513.

[201] T. P. Ying, M. X. Wang, X. X. Wu, Z. Y. Zhao, Z. Z. Zhang, B. Q. Song, Y. C. Li, B. Lei, Q. Li, Y. Yu, E. J. Cheng, Z. H. An, Y. Zhang, X. Y. Jia, W. Yang, X. H. Chen, S. Y. Li, Phys. Rev. Lett. 2018, 121, 207003.

[202] L. K. Ma, B. Lei, N. Z. Wang, K. S. Yang, D. Y. Liu, F. B. Meng, C. Shang, Z. L. Sun, J. H. Cui, C. S. Zhu, T. Wu, Z. Sun, L. J. Zou, X. H. Chen, arXiv:1808.06051

[203] K. Ueno, H. Shimotani, H. Yuan, J. Ye, M. Kawasaki, Y. Iwasa, J. Phys. Soc. Jpn. 2014, 83, 032001.

[204] S. Das Sarma, S. Adam, E. H. Hwang, E. Rossi, Rev. Mod. Phys. 2011, 83, 407.

[205] A. H. Castro Neto, F. Guinea, N. M. R. Peres, K. S. Novoselov, A. K. Geim, Rev. Mod. Phys. 2009, 81, 109.

[206] Y. Cao, V. Fatemi, S. Fang, K. Watanabe, T. Taniguchi, E. Kaxiras, P. Jarillo-Herrero, Nature 2018, 556, 43.

[207] Y. Cao, V. Fatemi, A. Demir, S. Fang, S. L. Tomarken, J. Y. Luo, J. D. Sanchez-Yamagishi, K. Watanabe, T. Taniguchi, E. Kaxiras, R. C. Ashoori, P. JarilloHerrero, Nature 2018, 556, 80.

[208] C. Xu, L. Balents, Phys. Rev. Lett. 2018, 121, 087001.

[209] S. Wu, V. Fatemi, Q. D. Gibson, K. Watanabe, T. Taniguchi, R. J. Cava, P. Jarillo-Herrero, Science 2018, 359, 76 .

[210] V. Fatemi, S. Wu, Y. Cao, L. Bretheau, Q. D. Gibson, K. Watanabe, T. Taniguchi, R. J. Cava, P. JarilloHerrero, Science 2018, 362, 926.

[211] E. Sajadi, T. Palomaki, Z. Fei, W. Zhao, P. Bement, C. Olsen, S. Luescher, X. Xu, J. A. Folk, D. H. Cobden, Science 2018, 362, 922.

[212] R. A. Klemm, A. Luther, M. R. Beasley, Phys. Rev. B $1975,12,877$.

[213] R. M. Lutchyn, E. P. A. M. Bakkers, L. P. Kouwenhoven, P. Krogstrup, C. M. Marcus, Y. Oreg, Nat. Rev. Mater. 2018, 3, 52.

[214] J. Alicea, Phys. Rev. B 2010, 81, 125318.

[215] R. M. Lutchyn, J. D. Sau, S. Das Sarma, Phys. Rev. Lett. 2010, 105, 077001.

[216] Y. Oreg, G. Refael, F. von Oppen, Phys. Rev. Lett. 2010, 105, 177002.

[217] A. Das, Y. Ronen, Y. Most, Y. Oreg, M. Heiblum, H. Shtrikman, Nat. Phys. 2012, 8, 887.

[218] K. T. Law, P. A. Lee, T. K. Ng, Phys. Rev. Lett. 2009, 103, 237001.

[219] J. D. Sau, S. Tewari, R. M. Lutchyn, T. D. Stanescu, S. Das Sarma, Phys. Rev. B 2010, 82, 214509.

[220] H. O. H. Churchill, V. Fatemi, K. Grove-Rasmussen, M. T. Deng, P. Caroff, H. Q. Xu, C. M. Marcus, Phys. Rev. B 2013, 87, 241401.

[221] M. T. Deng, C. L. Yu, G. Y. Huang, M. Larsson, P. Caroff, H. Q. Xu, Nano Lett. 2012, 12, 6414.

[222] S. M. Albrecht, A. P. Higginbotham, M. Madsen, F. Kuemmeth, T. S. Jespersen, J. Nygård, P. Krogstrup, C. M. Marcus, Nature 2016, 531, 206.

[223] D. Goldhaber-Gordon, H. Shtrikman, D. Mahalu, D. Abusch-Magder, U. Meirav, M. A. Kastner, Nature 1998, 391, 156.

[224] S. M. Cronenwett, H. J. Lynch, D. Goldhaber-Gordon, L. P. Kouwenhoven, C. M. Marcus, K. Hirose, N. S. Wingreen, V. Umansky, Phys. Rev. Lett. 2002, 88, 226805 . 
[225] L. P. Rokhinson, L. N. Pfeiffer, K. W. West, Phys. Rev. Lett. 2006, 96, 156602.

[226] L. P. Rokhinson, X. Liu, J. K. Furdyna, Nat. Phys. 2012, 8, 795 .

[227] B. Sacépé, J. B. Oostinga, J. Li, A. Ubaldini, N. J. G. Couto, E. Giannini, A. F. Morpurgo, Nat. Commun. 2011, 2, 575 .

[228] F. Qu, F. Yang, J. Shen, Y. Ding, J. Chen, Z. Ji, G. Liu, J. Fan, X. Jing, C. Yang, L. Lu, Sci. Rep. 2012, 2, 339.

[229] F. Yang, F. Qu, J. Shen, Y. Ding, J. Chen, Z. Ji, G. Liu, J. Fan, C. Yang, L. Fu, L. Lu, Phys. Rev. B 2012, 86, 134504 .

[230] J. R. Williams, A. J. Bestwick, P. Gallagher, S. S. Hong, Y. Cui, A. S. Bleich, J. G. Analytis, I. R. Fisher, D. Goldhaber-Gordon, Phys. Rev. Lett. 2012, 109, 056803.

[231] M. Veldhorst, M. Snelder, M. Hoek, T. Gang, V. K. Guduru, X. L. Wang, U. Zeitler, W. G. van der Wiel, A. A. Golubov, H. Hilgenkamp, A. Brinkman, Nat. Mater. 2012, 11, 417.

[232] S. Cho, B. Dellabetta, A. Yang, J. Schneeloch, Z. Xu, T. Valla, G. Gu, M. J. Gilbert, N. Mason, Nat. Commun. 2013, 4, 1689.

[233] J. B. Oostinga, L. Maier, P. Schüffelgen, D. Knott, C. Ames, C. Brüne, G. Tkachov, H. Buhmann, L. W. Molenkamp, Phys. Rev. X 2013, 3, 021007.

[234] A. D. K. Finck, C. Kurter, Y. S. Hor, D. J. Van Harlingen, Phys. Rev. X 2014, 4, 041022.

[235] Q. L. He, L. Pan, A. L. Stern, E. C. Burks, X. Che, G. Yin, J. Wang, B. Lian, Q. Zhou, E. S. Choi, K. Murata, X. Kou, Z. Chen, T. Nie, Q. Shao, Y. Fan, S.-C. Zhang, K. Liu, J. Xia, K. L. Wang, Science 2017, 357, 294.

[236] E. Wang, H. Ding, A. V. Fedorov, W. Yao, Z. Li, Y.F. Lv, K. Zhao, L.-G. Zhang, Z. Xu, J. Schneeloch, R. Zhong, S.-H. Ji, L. Wang, K. He, X. Ma, G. Gu, H. Yao, Q.-K. Xue, X. Chen, S. Zhou, Nat. Phys. 2013, 9, 621.

[237] M.-X. Wang, C. Liu, J.-P. Xu, F. Yang, L. Miao, M.Y. Yao, C. L. Gao, C. Shen, X. Ma, X. Chen, Z.-A. Xu, Y. Liu, S.-C. Zhang, D. Qian, J.-F. Jia, Q.-K. Xue, Science 2012, 336, 52.

[238] L. Fang, C. C. Stoumpos, Y. Jia, A. Glatz, D. Y. Chung, H. Claus, U. Welp, W.-K. Kwok, M. G. Kanatzidis, Phys. Rev. B 2014, 90, 020504.

[239] G. A. Wiegers, Prog. Solid State Chem. 1996, $24,1$.

[240] H. Bai, X. Yang, Y. Liu, M. Zhang, M. Wang, Y. Li, J. Ma, Q. Tao, Y. Xie, G.-H. Cao, Z.-A. Xu, J. Phys.Condes. Matter 2018, 30, 355701.
[241] Y. Saito, Y. Nakamura, M. S. Bahramy, Y. Kohama, J. Ye, Y. Kasahara, Y. Nakagawa, M. Onga, M. Tokunaga, T. Nojima, Y. Yanase, Y. Iwasa, Nat. Phys. 2016, 12, 144 .

[242] X. Xi, Z. Wang, W. Zhao, J.-H. Park, K. T. Law, H. Berger, L. Forró, J. Shan, K. F. Mak, Nat. Phys. 2016, 12, 139.

[243] E. O. Wrasse, T. M. Schmidt, Nano Lett. 2014, 14, 5717.

[244] J. Liu, X. Qian, L. Fu, Nano Lett. 2015, 15, 2657.

[245] J. Liu, T. H. Hsieh, P. Wei, W. Duan, J. Moodera, L. Fu, Nat. Mater. 2014, 13, 178.

[246] W. Wan, Y. Yao, L. Sun, C.-C. Liu, F. Zhang, Adv. Mater. 2017, 29, 1604788.

[247] E. J. H. Lee, X. Jiang, R. Aguado, G. Katsaros, C. M. Lieber, S. De Franceschi, Phys. Rev. Lett. 2012, 109, 186802.

[248] J. Liu, A. C. Potter, K. T. Law, P. A. Lee, Phys. Rev. Lett. 2012, 109, 267002.

[249] D. I. Pikulin, J. P. Dahlhaus, M. Wimmer, H. Schomerus, C. W. J. Beenakker, New J. Phys. 2012, 14, 125011.

[250] G. Kells, D. Meidan, P. W. Brouwer, Phys. Rev. B 2012, 86, 100503.

[251] S. Nadj-Perge, I. K. Drozdov, J. Li, H. Chen, S. Jeon, J. Seo, A. H. MacDonald, B. A. Bernevig, A. Yazdani, Science 2014, 346, 602.

[252] D. J. Clarke, J. Alicea, K. Shtengel, Nat. Phys. 2014, 10, 877.

[253] P. San-Jose, J. L. Lado, R. Aguado, F. Guinea, J. Fernández-Rossier, Phys. Rev. X 2015, 5, 041042.

[254] P. Zhang, K. Yaji, T. Hashimoto, Y. Ota, T. Kondo, K. Okazaki, Z. Wang, J. Wen, G. D. Gu, H. Ding, S. Shin, Science 2018, 360, 182.

[255] G. Bian, T.-R. Chang, R. Sankar, S.-Y. Xu, H. Zheng, T. Neupert, C.-K. Chiu, S.-M. Huang, G. Q. Chang, I. Belopolski, D. S. Sanchez, M. Neupane, N. Alidoust, C. Liua, B. K. Wang, C.-C. Lee, H.-T. Jeng, C. L. Zhang, Z. J. Yuan, S. Jia, A. Bansil, F. C. Chou, H. Lin, M. Z. Hasan, Nat. Commun. 2016, 7, 10556.

[256] S.-Y. Guan, P.-J. Chen, M.-W. Chu, R. Sankar, F. Chou, H.-T. Jeng, C.-S. Chang, T.-M. Chuang, Sci. Adv. 2016, 2, e1600894.

[257] T. Zhang, Y. Jiang, Z. Song, H. Huang, Y. He, Z. Fang, H. Weng, C. Fang, Nature 2019, 566, 475.

[258] F. Tang, H. C. Po, A. Vishwanath, X. Wan, Nature 2019, 566, 486.

[259] M. G. Vergniory, L. Elcoro, C. Felser, B. A. Bernevig, Z. Wang, Nature 2019, 566, 480. 